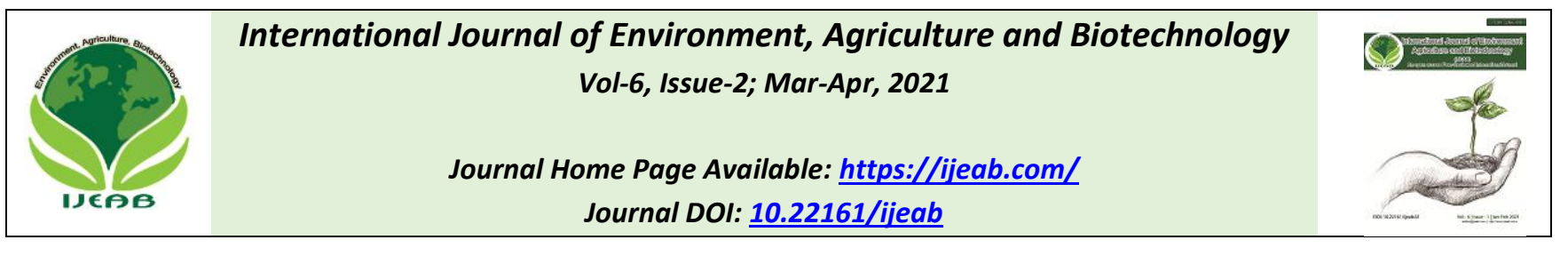

\title{
Prediction of High-Risk Probability Areas under Current and Future Climate Scenarios in India for the Establishment of Fall Armyworm
}

\author{
Dr. K. Susheela, K. A. Sai Swaroop and Dr. Alice R.P. Sujeetha
}

National Institute of Plant Health Management, Rajendranagar, Hyderabad, India

Received: 11 Dec 2020; Received in revised form: 15 Feb 2021; Accepted: 03 Mar 2021; Available online: 17 Mar 2021 (C)2021 The Author(s). Published by Infogain Publication. This is an open access article under the CC BY license (https://creativecommons.org/licenses/by/4.0/).

\begin{abstract}
The Fall Armyworm (FAW) or Spodoptera frugiperda, is an endemic and agriculturally important insect pest in tropical and subtropical regions of the Americas causing severe impact estimated at millions of dollars. FAW has been recently identified for the first time in India and is also a first record in Asia threatening the food security and livelihoods of millions of farmers. The insects are affected by climatic factors, and climate change may affect geographical distribution, abundance, growth rate, survival, mortality, number of generations per year and other characteristics. These climate change effects on insects are difficult to project due to complex interaction among insects, hosts and predators. Moreover, agricultural pest management may become more challenging under future climate change and variation. The present study aims to project the impact of climate change on future suitability for the expansion of FAW as well as highlight the high risk probability areas due to the pest using the historical and future climatic conditions. The modelling was carried out using CLIMEX model, GIS, the known distribution of the species and the CliMond meterological database. The analysis has indicated high climatic suitability for FAW occurrence in India with Eco-climatic Index (EI) values above 20. Further, the high risk probability areas for the FAW establishment up to district level were also identified for the major maize growing states. The areas where the pest is currently reported in the country are coinciding with the predicted potential areas in India validating the current analysis. The analysis using two general circulation models (GCMS), CSIRO MK3.0 and MIROC-H, for 2030 and 2050 under the A2 Special Report on Emissions Scenarios (SRES) indicated the possible reduction of climatically suitable areas for the FAW establishment in India. This kind of analysis assessing the possible impacts of FAW under future climate conditions is essential for the future economic production of crops.
\end{abstract}

Keywords-Fall army worm, Spodoptera frugiperda, Endangered areas, High Risk Probability, Ecoclimatic index (EI), CLIMEX.

\section{INTRODUCTION}

The fall armyworm, Spodoptera frugiperda (J.E.Smith) is an agriculturally important insect of more than 80 plant species, causing damage to economically important cultivated cereals such as maize, rice, sorghum, and also to vegetable crops and cotton with a particular preference for maize, a main staple crop around the world threatening food security and biosecurity. This highly-destructive and invasive pest has been seen in the Americas since several decades, but its prevalence outside was noted for the first time in West Africa in early 2016 (IITA 2016; IPPC 2016), and it has subsequently been recorded in most subSaharan countries (ACAPS 2017). It has spread to 44 countries across the continent, barring North Africa (CIMMYT 2018). Sightings of damage to maize crops in India due to fall armyworm mark the first report of the pest in Asia in 2018. In India it was reported for the first time from Karnataka (ICAR-NBAIR, 2018a) and Andhra 
Pradesh (EPPO, 2018). The pest has also been reported in Telangana, Tamil Nadu, Maharashtra and Gujarat.

Fall armyworm (FAW) causes major damage to economically important crops. In sub-Saharan Africa, where fall armyworm is devastating maize crops, estimates indicate 13.5 million tons of maize valued at $\$ 3$ billion are at risk in 2017-2018, which is equivalent to over 20 percent of total production for the region (CABI, April 2017). In America, FAW is listed as one of the major pest of maize and causing severe economic losses, with infection level up to $70 \%$. According to the International Maize and Wheat Improvement Centre (CIMMYT) at Mexico, FAW has, over the last two years, damaged more than 1.5 million hectares of Africa's maize crop. (Carolyn Cowan, Jennifer Johnson / August 13, 2018, CIMMYT). Moreover, FAW has suddenly become a major pest in Kenya, causing losses of about a third of the annual maize production, estimated at about 1 million tonnes (HugoDe Groote et al 2020).

Fall Armyworm is native to tropical and subtropical regions. However, it may also be found in temperate regions (Luginbill 1928; Sparks 1979; Clark et al. 2007). FAW does not possess a capability to enter diapause, due to this; FAW cannot survive extended periods of freezing temperature but must migrate northwards each spring if it is to re-infest cropping areas in temperate regions (Luginbill 1928).

The life cycle, behaviour, survival and spread of insects are affected by climate factors, especially temperature, which has a strong influence on all ectothermic organisms (Rosenzweig et al. 2001; Fhrer 2003; Diffen). Outbreaks of FAW are closely related to climatic conditions, and migrant adults can move northwards up to 483 $\mathrm{km} /$ generations with good winter and spring conditions (Sparks 1979). Climate change will have different effects on insects, directly impacting their life cycle or indirectly impacting hosts or predators (Cannon 1998; Patterson et al. 1999; Bale et al. 2002). Some of the expected effects are changes in geographic range, growth rate, migration, host preferences, abundance, synchronization, survival, mortality, number of generation per year and others (Tauber et al. 1986; Parry et al. 1990).

Climate change effects on insects are difficult to project due to complex interaction among insects, hosts and predators. Some modelling techniques have been developed to forecast the species distribution viz., Classification and Regression Tree Analysis (CART), Logistic Multiple Regression (LMR), Regression Tree Analysis (RTA), Maximum Entropy (MAXENT) and Bioclimatic Variable (BIOCLIM). Nevertheless, CLIMatic IndEX (CLIMEX) is especially appropriate for projecting the effects of variations in climate change on insects, since the model matches the presence of a particular organism with ranges of temperature, moisture and climatic stresses. In contrast to other models, CLIMEX uses more than one limiting factor to project current and future suitability (Sutherst et al. 1995; Patterson et al. 1999; Crozier \& Dwyer 2006; Shabani \& Kotey 2016). It has been widely used to project the current and future potential distribution of different pest (Kriticos et al. 2015b).

The main aim of the current research is to predict the high risk probability areas based on historical climatological data (centred at 1975) derived from Climond: global climatologies for bioclimatic modelling developed by The Commonwealth Scientific and Industrial Research Organisation, Australian federal government agency. An attempt has also been made to project the impact of climate change on future suitability of India for FAW, based on current known distribution using CLIMEX modelling and biological data obtained from the literature.

\section{MATERIAL AND METHODS}

CLIMEX (version 4.0) is a computational tool for studying the effects of climatic conditions on species distribution and relative abundance. The CLIMEX Model is based on the assumption that we can infer the suitable climatic conditions for a target species when information about its habitat is known. The compare location application was used to project the current probable distribution and spread of FAW in India.

"Compare Locations," application can predict the potential geographical distribution of target species with regard to climatic conditions (Jung et al 2016; Kriticos and Leriche 2010; Kriticos et al 2015; Stephens et al 2007). The species information necessary for the model is a series of parameters that describes responses to temperature, moisture and climatic stresses along with long term meteorological database for the location under study. The model assumes that the known distribution of the species infers the climatic conditions in which it can survive. The model parameters are divided among the population growth indexes, stress indexes and the constraint values, such as the length of the growing season (degree day per generation, PDD), which may exclude the organism from a particular location.

Growth index describes favorable season based on temperature, soil moisture, radiation, substrate, amount of light exposed, and diapause ability, whereas stress index is calculated from four stress indices, i.e., cold stress (CS), heat stress, wet stress, and dry stress, all of which are factors that limit the species population (Byeon et al 2017; Hill et al 2014; Kriticos et al 2015). The results of 
CLIMEX Model are represented by the Ecoclimatic index (EI), which indicates the survival and growth of a species in many different locations based on the climate. Ecoclimatic index (EI) describes the level of favourability of a location for the particular organism to survive when the conditions are favourable. The EI, expressed as numbers between 0 (Unfavourable conditions) and 100 (ideal conditions), is calculated by multiplying growth index, stress index, and interaction stress index. EI values near 0 represents a location where the species has poor conditions for long-term survival, while EI values > 30 indicate remarkably good conditions for establishment and survival of the species, and values close to 100 represent perfect conditions for the species (Sutherst et al. 2007; Kriticos et al. 2015a). These perfect conditions are difficult to achieve in nature but can be obtained in laboratory experiments (Sutherst et al. 2007; Kriticos et al. 2007a). In this present paper/study, the EI categorization was $\mathrm{EI}=0=$ unsuitable, a region where the population does not persist; EI $=1-10=$ marginal, where the population has limited conditions to persist; EI $=10-20=$ medium, where the region can support large population; and EI > 20 = optimal, where the population has highly favourable conditions to persist. These categorizations were developed by taking into account previous studies and the information in the user manual (Sutherst \& Maywald 1985; Shabani et al. 2014; Kriticos et al 2015a;
NYZ RAMIREZ-CABRAL et al 2017; Hannalene du Plessis et al. 2018).

For modelling the potential spread of FAW in the country (India), the current geographical distribution of FAW is necessary. The known geographical distribution was gathered from CABI (2020) and literature resources (Ramirez Cabral et al. 2017 and Westbrook et al. 2016)

\section{CLIMEX parameters:}

CLIMEX (Sutherst and Maywald 1985; Kriticos et al. 2015) uses a visual, iterative process to fit the stress parameters. In the present study, stress parameters that limit the species suitability have been taken from two different hypothesis of N.Y. Z. Ramirez - Cabral et al., and Hannalene Du Plessis et al. The stress parameters of both hypotheses have been tested to ensure the similarity of the generated potential geographic distribution with the species known geographic distribution. The hypothesis of Hannalene Du Plessis et al., fitting stress parameters appears to be more matching with the species known geographic distribution specified in CABI, 2020. Hence, the above hypothesis has been chosen to run the model and generate the CLIMEX simulation consistent with the current distribution of FAW. CLIMEX parameter values used for modelling the distribution of FAW is given in the table 1 .

Table 1: CLIMEX parameter values for modelling the distribution of FAW

\begin{tabular}{|c|c|c|}
\hline Parameter & Description & Values \\
\hline \multicolumn{3}{|l|}{ Moisture } \\
\hline SM0 & Lower soil moisture threshold & 0.15 \\
\hline SM1 & Lower optimal soil moisture & 0.8 \\
\hline SM2 & Upper optimal soil moisture & 1.5 \\
\hline SM3 & Upper soil moisture threshold & 2.5 \\
\hline \multicolumn{3}{|c|}{ Temperature } \\
\hline DV0 & Lower temperature threshold & $12{ }^{\circ} \mathrm{C}$ \\
\hline DV1 & Lower optimal temperature & $25^{\circ} \mathrm{C}$ \\
\hline DV2 & Upper optimal temperature & $30^{\circ} \mathrm{C}$ \\
\hline DV3 & Upper temperature threshold & $39^{\circ} \mathrm{C}$ \\
\hline \multicolumn{3}{|l|}{ Cold Stress } \\
\hline TTCS & Cold stress temperature threshold & $12{ }^{\circ} \mathrm{C}$ \\
\hline THCS & Cold stress accumulation rate & 0.001 week $^{-1}$ \\
\hline \multicolumn{3}{|l|}{ Heat Stress } \\
\hline TTHS & Heat stress temperature threshold & $39^{\circ} \mathrm{C}$ \\
\hline THHS & Heat stress accumulation rate & 0.005 week $^{-1}$ \\
\hline Dry Stress & & \\
\hline
\end{tabular}




\begin{tabular}{|l|l|l|}
\hline $\begin{array}{l}\text { SMDS } \\
\text { HDS }\end{array}$ & $\begin{array}{l}\text { Soil moisture dry stress threshold } \\
\text { Dry stress accumulation rate }\end{array}$ & $\begin{array}{l}0.1 \\
-0.005 \text { week }^{-1}\end{array}$ \\
\hline Wet Stress & \\
\hline $\begin{array}{l}\text { SMWS } \\
\text { HWS }\end{array}$ & Soil moisture wet stress threshold & 2.5 \\
\hline Threshold Annual Heat Sum & Wet stress accumulation rate & 0.002 week $^{-1}$ \\
\hline PDD & Minimum degree day sum needed to complete a generation & $600^{\circ} \mathrm{C}$ \\
\hline
\end{tabular}

Once the parameters were included, the CLIMEX output was exported to Arch Map GIS and maps of climate suitability (EI) for establishment of FAW were generated for India and its individual states (Source of GIS maps: Indian Space Research Organisation, Dept. of Space, Govt. of India, Balanagar, Hyderabad). Validation was also done with the existing locations of occurrence in India.

An attempt was also made to project the future climatic suitability for the years 2030 and 2050. The current suitability was modelled with the Climond 10' baseline data, gridded historical climate data and the average 196190 baseline period in Climex format (Kriticos et al. 2012b). Among the different future climate scenarios (A1B, A2, B1 and B2), A2 scenario was chosen to model the future climatic suitability owing to its emission scenario with actual $\mathrm{CO}_{2}$ emissions, increase in greenhouse gases and agricultural productivity. Two Global Climate Models (GCM's) were used to model the future climatic suitability for FAW with future data, viz., CSIRO MK3.0, from the Common Wealth Scientific and Industrial Research Organisation from Australia (Gordon et al. 2010), and MIROC-H from the Centre for Climate Research in Tokyo, Japan (Shiogama et al. 2010). These GCMs were chosen because of the availability of climatic variables required for CLIMEX.

\section{OBSERVATIONS:}

The parameter values from the hypothesis of Hannalene Du Plessis et al., relevant to species biology under "Wet Tropical" species condition were considered to project potential distribution of FAW. The parameters are explained in detail below-

- The Moisture Index is estimated from a hydrological model based on soil moisture, rainfall and evaporation, with information of the previous and current week. This index denotes the response of the organism to soil moisture and assumes it to be constant over a $24 \mathrm{~h}$ period. A value of zero indicates no soil moisture and no growth. The parameters are SM0 = lower soil moisture threshold, SM1 = lower optimal soil moisture, SM2 = upper optimal soil moisture and SM3 = upper soil moisture threshold (Sutherst et.al 2007). Because FAW depends on a host survival, the insect will be unable to survive if the crop or plant is dead. Based on this assumption, the SM0 was set at 0.15 , the wilting point value most frequently used (Kriticos et al. 2014), which is almost in agreement with the SMDS. Moisture is known to affect the pupal stage, and excessive dryness retards emergence (Vickery 1929). Soil moisture parameters were set to biologically reasonable values. The soil moisture limit for growth was set to approximate permanent wilting point (Kriticos et al. 2003). The upper limit for optimal growth (SM2) was set to 2.5, acknowledging that $S$. frugiperda can tolerate conditions with substantial water-logging. The lower limit for optimal growth (SM1) was adjusted to provide appropriate suitability in marginally dry areas.

- The Temperature Index is one of the main components of the growth index. It denotes suitable temperature ranges in which the organism can live, with values ranging from 0 to 1. There are four parameters of this index: the lower temperature threshold (DV0), the lower optimum temperature (DV1), the upper optimum temperature (DV2) and the upper temperature threshold (DV3) (Sutherst et al. 2007). The lower temperature threshold for growth is $12^{\circ} \mathrm{C}$ which reflect the tropical distribution of $S$. frugiperda. The lower temperature for optimal growth is 25 ${ }^{\circ} \mathrm{C}$, the upper optimal temperature for growth is $30{ }^{\circ} \mathrm{C}$ (Simmons 1993), and the maximum temperature is $39^{\circ} \mathrm{C}$, near the threshold of $39.8^{\circ} \mathrm{C}$ reported by Valdez- Torres et al. (2012). Taking into account the above experimental results, the value for DV0 was set at $12^{\circ} \mathrm{C}$. DV1 and Dv2 were set as $25^{\circ} \mathrm{C}$ and $30{ }^{\circ} \mathrm{C}$ respectively.

- FAW does not diapause and cannot survive the winters in temperate areas (Luginbill 1928; 
Johnson 1987). Diapause was therefore not included in this model.

- $\quad$ The cold stress temperature threshold (TTCS) and the accumulation rate of this threshold (cold stress temperature rate, THCS) were used to project current suitability for FAW. A temperature threshold model of Cold Stress was used, with a $12^{\circ} \mathrm{C}$ threshold and a stress accumulation rate of 0.001 week $^{-1}$. CLIMEX can calculate the cold stress and exclude the survival of an insect when exposed to low temperatures (Sutherst et al. 2007). Although FAW does not tolerate prolonged and extreme cold periods, periods of mild cold and rainfall promote the abundance of the insect (Luginbill 1928; Westbrook et al. 2016).

- The heat stress temperature threshold (TTHS) and its accumulation rate (heat stress accumulation rate, THHS), were used to limit the survival of FAW at high temperatures. When the average maximum temperature is below TTHS, the heat stress is equal to zero (Sutherst et al 2007). The threshold of $39{ }^{\circ} \mathrm{C}$ is the same as the upper temperature limit for development.

- CLIMEX accumulates dry stress when soil moisture is lower than the dry stress threshold (SMDS). This stress is accumulated weekly and is multiplied the dry stress rate (HDS) (Sutherst et al 2007). There is evidence that during a dry season, few adult moths are trapped and the population peaks are delayed (Andrews 1988). The dry stress was set at $\mathrm{SMDS}=0.1$ to avoid the persistence of FAW. Dry Stress was given by using the lower soil moisture growth threshold and adjusting the rate to limit the distribution to tropical and subtropical regions where it has been reported.

- Wet stress accumulates when soil moisture exceeds the wet stress threshold (SMWS). Annual wet stress depends on the sum of weeks per year during which this stress occurs. Wet stress accumulates at a wet stress rate (Sutherst et al. 2007). A higher number of FAW moths have been recorded when rainfall is plentiful. While heavy rains reduce the population density of larvae in the early instars, this does not affect late instars or the adult stage (Luginbill 1928; Andrews 1988). The SMWS was set high at 1.5, with an accumulation rate of $0.002 /$ week $^{-1}$, to allow persistence throughout the known distribution.
- This parameter describes the necessary number of growing degree days to complete a generation. It acts as a constraint, limiting the organism according to the level of suitability of a specific location. If the minimum PDD is not met, the EI equal to 0 (Sutherst et al. 2007). Studies have reported that the life cycle takes between 38 and 62 calendar days to complete, depending on temperature and humidity (Vickery 1929; Sparks 1979). However, this is not an exact measurement in that it varies according to climatic zones. Growing degree days allow a more accurate measurement of the growth and development of insects during the growing season. Valdez-Torres et al. (2012) calculated the PDD for FAW at 504, with a temperature base $\left(\mathrm{T}_{\mathrm{b}}\right)$ of $8.7^{\circ} \mathrm{C}$, while another experiment used PDD $=559.1$, with $\mathrm{T}_{\mathrm{b}}=$ $10.9^{\circ} \mathrm{C}$ (Ramirez Garcia et al. 1987). In the current study, PDD was set at 600 , as per Ramirez Cabral et al. 2017, because $\mathrm{T}_{\mathrm{b}}$ is closer to DV0 i.e., $12^{\circ} \mathrm{C}$. This value is the time from egg to adult.

The resulting maps show areas that are likely to be at different level of risk of FAW as determined from the climatic suitability range (Fig 1). The current climate conditions modelled for FAW showed suitability for FAW occurrence in India.

- Tropical regions were found to be more suitable for its establishment which is similar to the observations of Luginbill 1928. High risk suitability areas with $\mathrm{EI}=>20$ for FAW occurrence in India are projected in Fig 2.

- Currently, infestation of FAW is reported in twelve states in India viz., Andhra Pradesh, Bihar, Chhattisgarh, Goa, Gujarat, Karnataka, Madhya Pradesh, Maharashtra, Odisha, Tamil Nadu, Telangana and West Bengal (CABI, 2020). The current analysis with Compare locations (Species 1) application has also indicated high climatic suitability in the above states with 20-95 EI values validating the present study (Fig 2).

- Climate is not the only factor limiting species geographical distribution (Brown et al., 1996). Host availability is also important in actual determination of potential geographic areas. Though, FAW is polyphagous attacking about 80 plant species, it is reported mainly on maize in India. Therefore, 12 major Maize growing areas viz., Andhra Pradesh, Bihar, Gujarat, Jharkhand, Karnataka, Madhya Pradesh, Maharashtra, Rajasthan, Tamil Nadu, Telangana, Uttar Pradesh 
and West Bengal are considered while predicting the climatically suitable potential areas showing EI values between 20 - 95 for FAW establishment and the potential areas were given up to Taluk level (Table 2).

- High risk suitability areas are mostly noticed in South India and North east India. High risk potential areas were noticed in eight major maize growing states viz., Andhra Pradesh, Bihar, Jharkhand, Karnataka, Madhya Pradesh, Maharashtra, Tamil Nadu and Telangana while risk potential was medium to low in states of Rajasthan, Gujarat and Uttar Pradesh.

- The probable areas for the establishment of FAW based on climatic suitability and presence of major host, i.e., Maize are projected in the Indian map (Fig 3), while the State maps are projected in Fig 5.

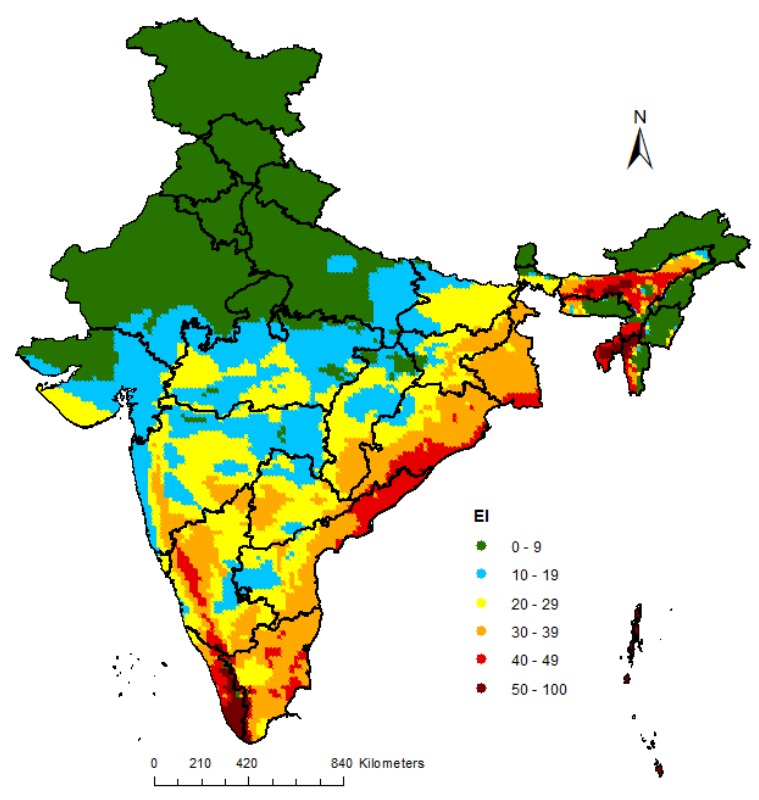

Fig 1: Different Risk probability areas in India for the Establishment of Fall army worm

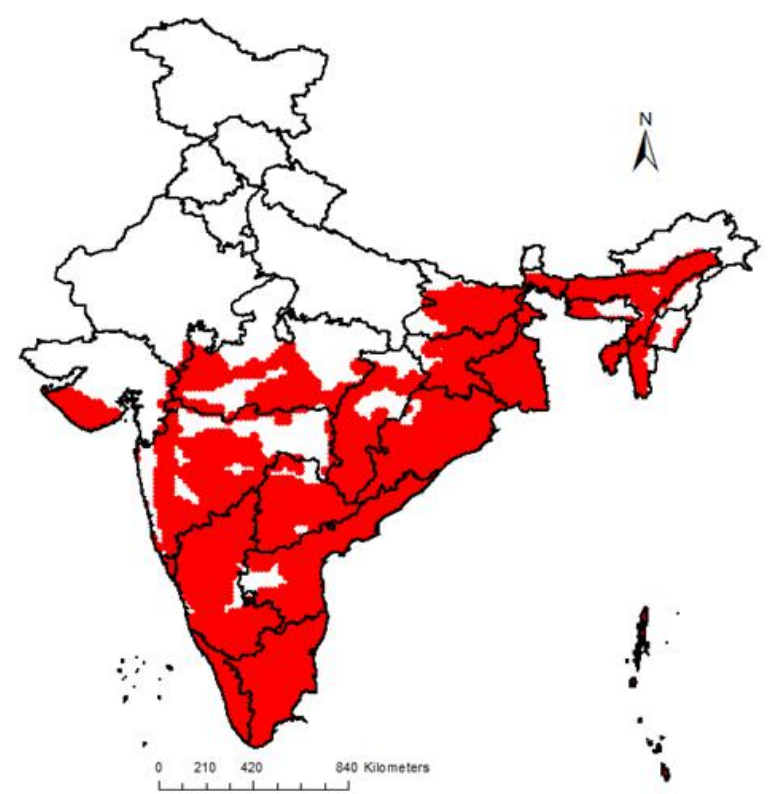

Fig 2: High Risk probability areas in India for the Establishment of Fall army worm 


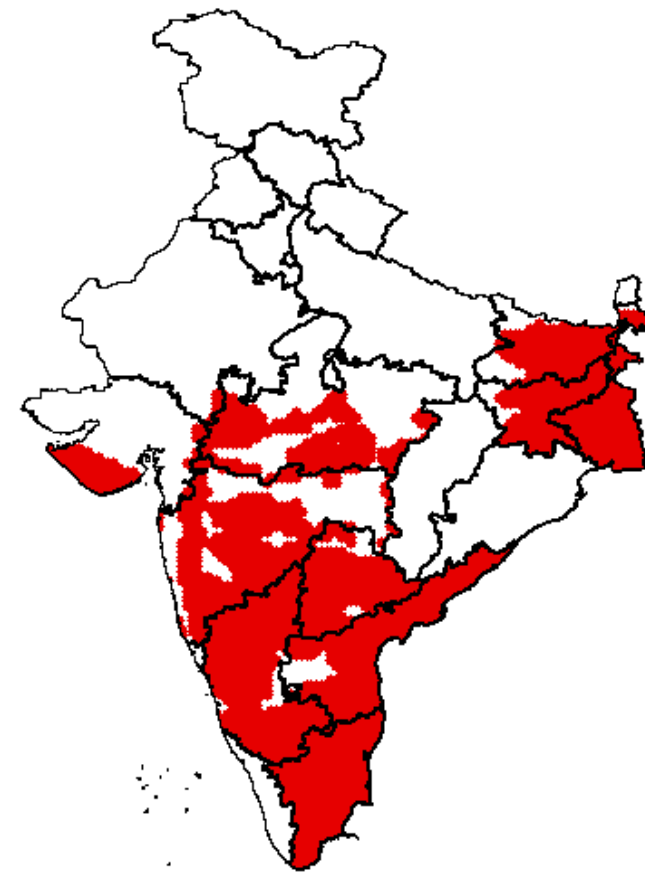

$0 \quad 230 \quad 480 \quad 920$ Kilometers
Fig 3: High Risk probability areas for the Establishment of Fall army worm in major Maize growing states in India

The CSIRO 2030, CSIRO 2050, MIROC 2030 and MIROC 2050 scenarios implemented under A2 emission scenario follow a similar pattern with different suitability with marked reduction by 2050 in the potential area for establishment of FAW (Fig 4a, 4b, 4c \& 4d). The potential area will decrease its suitability significantly under MIROC 2050 in southern India eliminating few states viz., Madhya Pradesh, Maharashtra, Telangana and Andhra Pradesh compared with the current scenario for FAW risk.

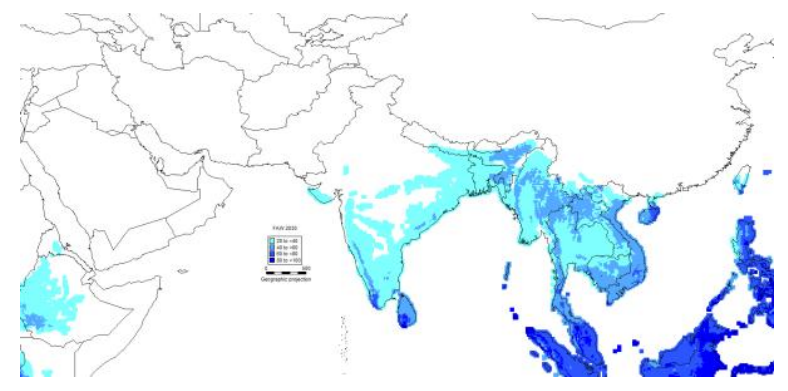

Fig 4a: CSIRO 2030

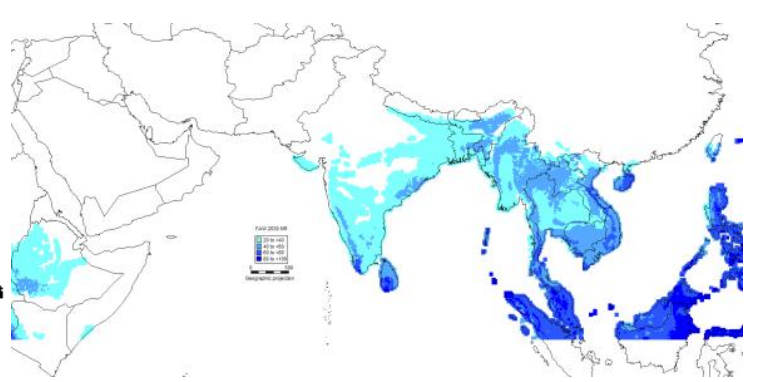

Fig 4b: MIROC 2030

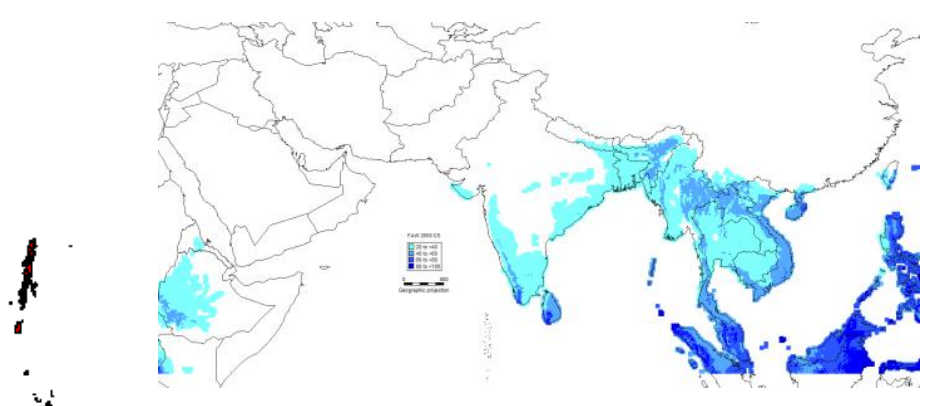

Fig 4c: CSIRO 2050

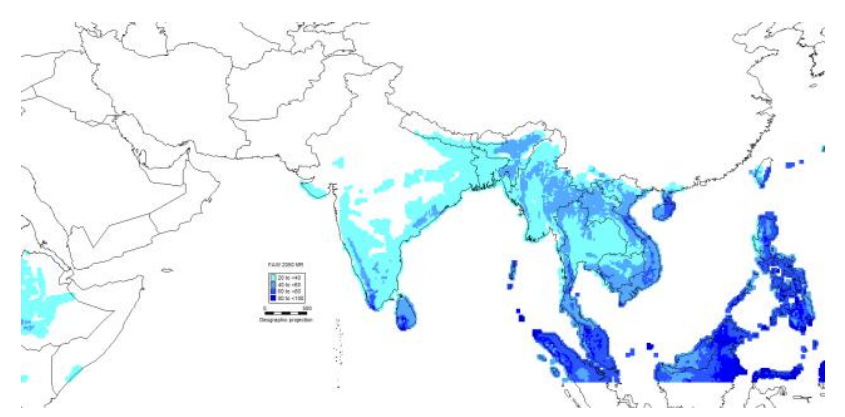

Fig 4d: MIROC 2050

\section{DISCUSSION}

The current FAW climate conditions projected for the model closely matches previous distributions, where FAW is listed as the major maize pest. In the present study, the reliability, accuracy and robustness of Climex model are reflected in high proportions from the validation area falling within the modelled area. The currently generated potential areas for fall army worm also include the recently infested areas in India thus validating the current prediction model. The maps generated based on Compare species 1 option appears to be more precise for the FAW species establishment. Hence, this option is recommended for better analysis subjected to the availability of the data on growth and stress parameters of the species. The data 
on the potential districts and their respective taluks for the establishment of pests is also generated using Arc GIS.

High suitability was shown in tropical wet and dry regions in India i.e., South Indian states as per Koppen classification (Köppen, W etal., 1884). The marginal suitability projected by the model in few humid subtropical regions as in the case of Bihar, Jharkhand and Madhya Pradesh could be due to the fact that FAW occurs seasonally by migration from warm climates to subtropical regions. This migration has been estimated at a rate of movement of $40 \mathrm{~km} / g$ eneration in years with favourable climate conditions (Westbrooks \& Sparks 1986) that allow FAW to migrate.

The modelling carried out under the $\mathrm{A} 2$ scenario, predicts an increase in temperature for 2030 and 2050. These small increases in temperature are likely to change the current suitability of the climate for FAW in India leading to the reduction of potential area especially in Southern India. In tropical areas, insects are close to their thermal tolerance limits and so, small increase in temperature may reduce or prevent their survival. Conversely, the increase in temperature in cold places may enhance the insect fitness and survival indicating that the impact in tropical regions may be more drastic than in temperate regions and that the benefit of warmer temperatures will depend on the temperature sensitivity of the species (Helmuth et al. 2002, Deutsch et al. 2008, Tewksbury et al. 2008). Further, polyphagous pests adapt better to climate change due to their phenotypic and genotypic plasticity and may even feed on the hosts of lower quality when preferential hosts are not available (Sparks, 1979, Randall 1986, Bale et al 2002). The above results are consistent with the studies of Svobodova et al. 2014 and Ramirez cabral et al. 2017 explaining the reduction in suitable areas due to increased temperatures and reduced moisture levels.

\section{CONCLUSION AND WAY FORWARD}

The FAW is polyphagous pest with many economically important hosts majorly attacking maize in India. Predicting the climatically suitable areas in India is essential to protect the future production of both staple and non-staple crops, since FAW attacks both. With this information, farmer, policy makers and government could implement adaption measures, such as the new technology, new varieties and management practices to overcome the impacts of FAW on important economic crops.

Pest management in agriculture may be more challenging under future climate and variability. The risk maps generated has application for mainly three types of personnel: pest survey specialists, program managers, and risk analysts for implementing surveys, for allocation of resources and to assess the potential establishment for high-risk pathways, commodities, or pests, respectively. Risk maps may also be useful in delimiting surveys or for managing pest eradications.

The results can be used to help guide pest risk assessments by the National Plant Protection Organization (NPPO), effective monitoring and surveillance of the unintentional introductions of this pest via trade from currently infested countries, and, policy makers and trade negotiators in making science-based decisions. In addition, India which is dealing with a relatively recent introduction and spread of fall army worm, the generated maps can be used to identify areas most at risk of the expansion of this pest. Efforts can be coordinated and concentrated strategically across susceptible areas to stem the incursion. An additional potential application of these maps is for Regional Central Integrated Pest Management Centres (CIPMCs) in the identification of areas most suitable for area-wide pest suppression or eradication. Areas with established populations of pests which are on the extreme margins of climate suitability can be targeted as the most likely locations for these suppression and eradication efforts.

The current modelling approach was carried out using only the climate data, which ignore potential genetic changes in species and adaption to new climatic conditions (Bradshaw \& Holzapfel 2006). Future studies at global and regional levels are essential for better understanding of the FAW risk. 
Table 2: Major Maize growing states with high risk probability areas for the establishment of Fall Armyworm with EI=>20.

\begin{tabular}{|c|c|c|}
\hline $\begin{array}{l}\text { Major Maize } \\
\text { Growing } \\
\text { States }\end{array}$ & Potential Districts & Potential Taluks \\
\hline \multirow{13}{*}{$\begin{array}{l}\text { Andhra } \\
\text { Pradesh }\end{array}$} & Anantapur & Gudibanda, Hindupur, Kadiri, Penukonda, Madakasira \\
\hline & Chittoor & $\begin{array}{l}\text { Satyavedu, Puttur, Chandragiri, Chittoor, Srikalahasti, Palamaner, Punganur, } \\
\text { Kuppam, Vayalpad, Madanapalle }\end{array}$ \\
\hline & Cuddapah & Rajampet, Rayachoti, Sidhout, Badvel, Proddatur, Cuddapah \\
\hline & East Godavari & $\begin{array}{l}\text { Prathipadu, Ellavaram, Razole, Amalapuram, Mummidivaram, Kakinada } \\
\text { (Urban), Peddapuram, Rampachodavaram, Kothapeta, Kakinada (Urban), } \\
\text { Peddapuram, Tuni, Ramachandrapuram1, Rajahmundry (Urban). }\end{array}$ \\
\hline & Guntur & $\begin{array}{l}\text { Repalle, Tenali, Bapatla, Guntur, Narasaraopet , Sattenapalle, Vinukonda, } \\
\text { Gurazala }\end{array}$ \\
\hline & Krishna & $\begin{array}{l}\text { Machilipatnam, Avanigadda, Kaikalur, Gudivada, Nuzvid, Tiruvuru, } \\
\text { Vijayawada (Urban), Gannavaram, Nandigama, Jaggayyapeta }\end{array}$ \\
\hline & Kurnool & $\begin{array}{l}\text { Nandyal, Atmakur2, Allagadda, Emmiganuru, } \\
\text { Nandikotkur, Dhone, Kurnool }\end{array}$ \\
\hline & Nellore & $\begin{array}{l}\text { Sullurpeta, Venkatagiri, Gudur1, Nellore, Kavali, Rapur, Atmakur3, Kovur, } \\
\text { Rapur, Udayagiri }\end{array}$ \\
\hline & Prakasam & $\begin{array}{l}\text { Markapur, Giddalur, Addanki, Kandukur, Darsi, Chirala, Ongole, Kanigiri, } \\
\text { Podili }\end{array}$ \\
\hline & Srikakulam & $\begin{array}{l}\text { Palakonda, Pathapatnam, Srikakulam, Sompeta, Ichchapuram, Tekkali, } \\
\text { Narasannapeta }\end{array}$ \\
\hline & Visakhapatnam & $\begin{array}{l}\text { Chodavaram, Ananthagiri, Chintapalle, Narsipatnam, Anakapalle, } \\
\text { Visakhapatnam (Urban), Paderu, Bheemunipatnam, Yelamanchili. }\end{array}$ \\
\hline & Vizianagaram & $\begin{array}{l}\text { Srungavarapukota, Salur, Parvathipuram, Vizianagaram, Chipurupalle, } \\
\text { Pusapatirega }\end{array}$ \\
\hline & West Godavari & $\begin{array}{l}\text { Narsapuram, Polavaram, Bhimavaram, Tanuku, Tadepalligudem, Kovvur, } \\
\text { Tadepalligudem, Eluru. }\end{array}$ \\
\hline \multirow[t]{6}{*}{ Telangana } & Adilabad & Asifabad, Utnoor, Luxettipet, Boath, Nirmal, Khanapur, Adilabad, Mudhole. \\
\hline & Hyderabad & Hyderabad \\
\hline & Karimnagar & $\begin{array}{l}\text { Sircilla, Huzurabad, Karimnagar, Sircilla, Karimnagar, Manthani, Medipalle, } \\
\text { Jagtial, Sultanabad }\end{array}$ \\
\hline & Khammam & Burgampahad, Kothagudem1, Madhira, Yellandu, Nugur, Khammam (Urban). \\
\hline & Mahbubnagar & $\begin{array}{l}\text { Achampet, Farooqnagar, Kodangal, Kollapur, Mahbubnagar, Kalwakurthy, } \\
\text { Nagarkurnool, Makthal, Wanaparthy, Gadwal, Atmakur1, Alampur. }\end{array}$ \\
\hline & Medak & $\begin{array}{l}\text { Zahirabad, Narayankhed, Sangareddy, Gajwel, Andole, Siddipet, Narsapur, } \\
\text { Medak }\end{array}$ \\
\hline
\end{tabular}




\begin{tabular}{|c|c|c|}
\hline & Nalgonda & Bhongir, Devarakonda, Ramannapeta, Nalgonda, Suryapet, Huzurnagar \\
\hline & Nizamabad & $\begin{array}{l}\text { Kamareddy, Yellareddy, Madnoor, Armur, Banswada, Nizamabad, Armur, } \\
\text { Bodhan }\end{array}$ \\
\hline & Rangareddi & $\begin{array}{l}\text { Pargi, Tandur, Vicarabad, Chevella, Rajendranagar, Medchal, Ibrahimpatnam, } \\
\text { Hayathnagar }\end{array}$ \\
\hline & Warangal & Narsampet, Jangaon, Hanamkonda, Mulug1, Mahabubabad, Parkal \\
\hline Karnataka & Bagalkot & Badami, Bilgi, Jamkhandi, Hungund, Bagalkot, Mudhol \\
\hline & Bangalore & Anekal, Bangalore South, Bangalore North \\
\hline & Bangalore Rural & $\begin{array}{l}\text { Ramanagaram, Kanakapura, Magadi, Nelamangala, Devanahalli, Dod } \\
\text { Ballapur, } \\
\text { Channapatna, Hosakote. }\end{array}$ \\
\hline & Belgaum & $\begin{array}{l}\text { Sampgaon, Hukeri, Belgaum, Parasgad, Gokak, Chikodi, Ramdurg, Khanapur } \\
\text {, Raybag, Athni. }\end{array}$ \\
\hline & Bellary & Hadagalli, Sandur, Hospet, Kudligi, Hagaribommanahalli, Siruguppa \\
\hline & Bidar & Bidar, Homnabad, Bhalki, Aurad, Basavakalyan, Aurad \\
\hline & Bijapur & Bijapur, Basavana Bagevadi, Muddebihal, Sindgi, Indi. \\
\hline & Chamarajanagar & Kushtagi, Yelbarga, Koppal, Gangawati. \\
\hline & Chamaraja-nagar & Gundlupet, Kollegal, Yelandur, Chamrajnagar \\
\hline & Chikmagalur & Narasimharajapura, Tarikere, Chikmagalur, Kadur, Koppa, Mudigere, Sringeri \\
\hline & Chitradurga & Holalkere, Chitradurga, Hosadurga \\
\hline & Dakshina Kannada & Sulya, Puttur, Beltangadi, Bantval, Mangalore. \\
\hline & Davanagere & Honnali, Channagiri, Harpanahalli, Harihar, Davanagere, Jagalur. \\
\hline & Dharwad & Kalghatgi, Hubli, Dharwad, Navalgund \\
\hline & Gadag & Shirhatti, Mundargi, Gadag, Ron, Nargund. \\
\hline & Gulbarga & $\begin{array}{l}\text { Sedam, Chincholi, Shorapur, Gulbarga, Yadgir, Aland, Jevargi, Chitapur, } \\
\text { Shahpur, Afzalpur. }\end{array}$ \\
\hline & Hassan & $\begin{array}{l}\text { Arkalgud, Hassan, Belur, Alur, Belur, Hole Narsipur, Manjarabad, } \\
\text { Channarayapatna, Arsikere. }\end{array}$ \\
\hline & Haveri & Hangal, Shiggaon, Hirekerur, Haveri, Savanur, Ranibennur, Hirekerur \\
\hline & Kodagu & Virajpet, Somvarpet, Madikeri \\
\hline & Kolar & $\begin{array}{l}\text { Chik Ballapur, Gauribidanur, Sidlaghatta, Kolar, Chintamani, Bagepalli, } \\
\text { Malur, Bangarapet, Mulbagal, Gauribidanur }\end{array}$ \\
\hline & Mandya & $\begin{array}{l}\text { Maddur, Malavalli, Shrirangapattana, Krishnarajpet, Maddur, Pandavapura, } \\
\text { Nagamangala }\end{array}$ \\
\hline & Mysore & $\begin{array}{l}\text { Heggadadevankote, Piriyapatna, Hunsur, Nanjangud, Krishnarajanagara, } \\
\text { Tirumakudal Narsipur, Mysore }\end{array}$ \\
\hline & Raichur & Lingsugur, Devadurga, Raichur, Manvi, Sindhnur \\
\hline
\end{tabular}




\begin{tabular}{|c|c|c|}
\hline & Shimoga & Shimoga, Sorab, Bhadravati, Shikarpur, Sagar, Tirthahalli, Hosanagara \\
\hline & Tumkur & $\begin{array}{l}\text { Kunigal, Tumkur, Gubbi, Koratagere, Turuvekere, Tiptur, Chiknayakanhalli, } \\
\text { Madhugiri, Pavagada. }\end{array}$ \\
\hline & Udupi & Karkal, Udupi \\
\hline & Uttara Kannada & Haliyal, Mundgod, Yellapur, Supa, Sirsi, Siddapur, Ankola, Karwar, Kumta \\
\hline Tamil Nadu & Ariyalur & Udayarpalayam, Ariyalur \\
\hline & Chennai & Chennai \\
\hline & Coimbatore & $\begin{array}{l}\text { Coimbatore, Pollachi, Udumalaipettai, Mettupalayam, Avanashi, Palladam, } \\
\text { Udumalaipettai. }\end{array}$ \\
\hline & Cuddalore & Kattumannarkoil, Virudhachalam, Cuddalore, Chidambaram \\
\hline & Dharmapuri & Harur, Denkanikottai, Pennagaram, Palakkodu, Uthangarai, Krishnagiri \\
\hline & Dindigul & Kodaikanal, Palani, Dindigul, Natham, Nilakkottai, Vedasandur \\
\hline & Erode & Sathyamangalam, Bhavani, Perundurai, Dharapuram, Erode. \\
\hline & Kancheepuram & $\begin{array}{l}\text { Cheyyur, Sriperumbudur, Uthiramerur, Chengalpattu, Kancheepuram, } \\
\text { Tambaram }\end{array}$ \\
\hline & Kanniyakumari & Vilavancode, Thovala, Kalkulam \\
\hline & Karur & Kulithalai, Krishnarayapuram, Karur \\
\hline & Madurai & $\begin{array}{l}\text { Usilampatti, Melur, Madurai North, Vadipatti, } \\
\text { Thirumangalam }\end{array}$ \\
\hline & Nagapattinam & Vedaranyam, Mayiladuthurai, Tharangambadi, Sirkali \\
\hline & Namakkal & Namakkal, Rasipuram, Tiruchengode \\
\hline & Perambalur & Perambalur, Thuraiyur \\
\hline & Pudukkottai & Aranthangi, Pudukkottai, Alangudi, Thirumayam, Kulathur \\
\hline & Ramanathapuram & Tiruvadanai, Ramanathapuram, Paramakudi, Mudukulathur, Kamuthi \\
\hline & Salem & Salem, Attur, Omalur, Mettur, Sankari \\
\hline & Sivaganga & Tirupathur, Karaikkudi, Sivaganga, Devakottai, Ilayangudi. \\
\hline & Thanjavur & Pattukkottai, Peravurani, Orathanadu, Papanasam, Kumbakonam, Thanjavur \\
\hline & The Nilgiris & Udhagamandalam, Gudalur, Kotagiri \\
\hline & Theni & Uthamapalayam, Periyakulam \\
\hline & Thiruvallur & Tiruttani, Uthukkottai, Ambattur, Pallipattu, Ponneri, Gummidipoondi \\
\hline & Thiruvarur & Mannargudi, Nannilam, Thiruvarur \\
\hline & Thoothukkudi & $\begin{array}{l}\text { Kovilpatti, Sathankulam, } \\
\text { Srivaikuntam, Thoothukkudi }\end{array}$ \\
\hline & Tiruchirappalli & Manapparai, Lalgudi, Tiruchirappalli \\
\hline & Tirunelveli & $\begin{array}{l}\text { Tenkasi, Shenkottai, Ambasamudram, Sivagiri, Sankarankoil, Radhapuram, } \\
\text { Nanguneri, Tirunelveli, Palayamkottai }\end{array}$ \\
\hline
\end{tabular}




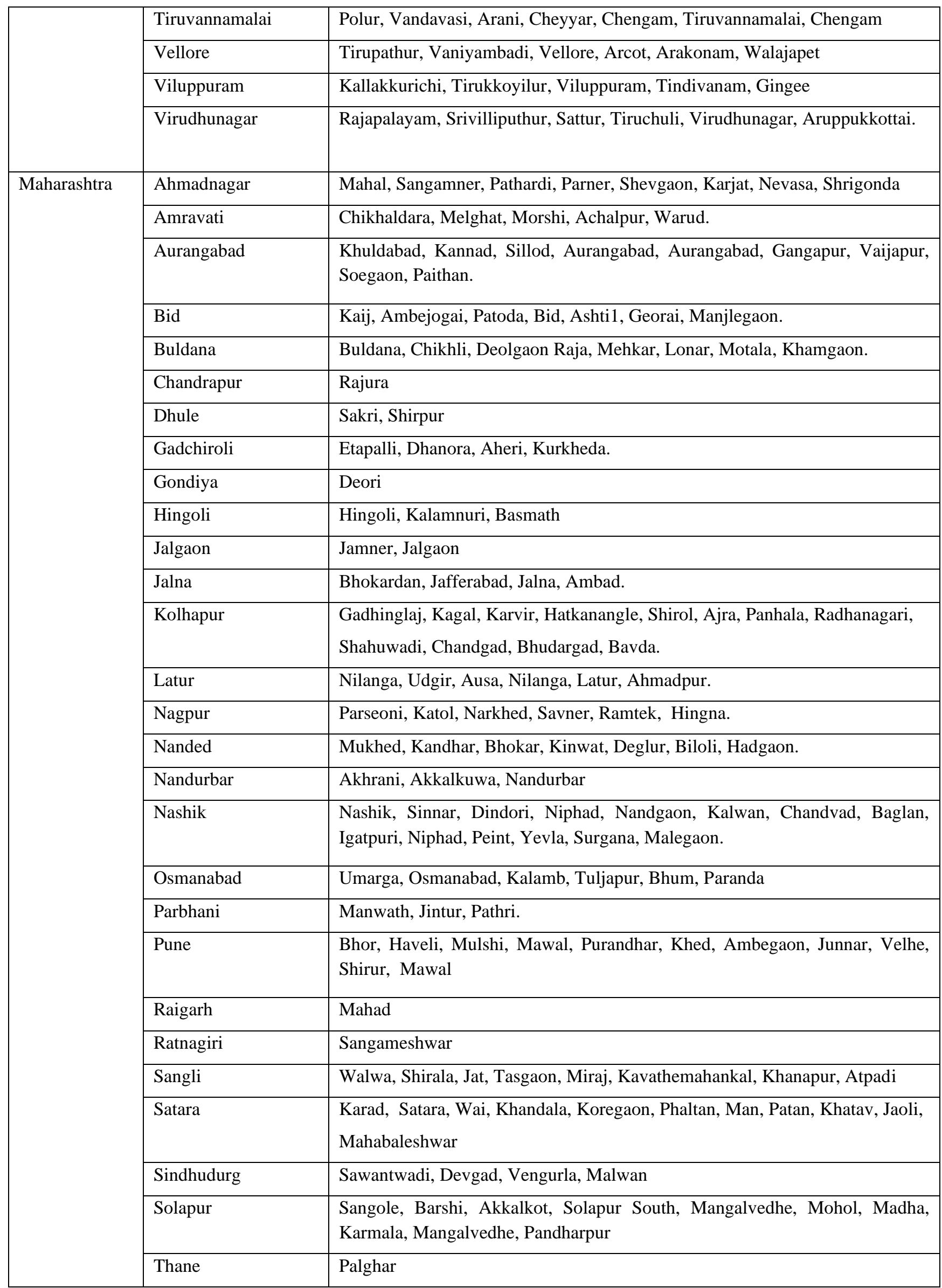




\begin{tabular}{|c|c|c|}
\hline & Wardha & Karanja \\
\hline & Washim & Risod, Malegaon, Mangrulpir, Manora \\
\hline & Yavatmal & Pusad, Umarkhed, Mahagaon \\
\hline \multirow{34}{*}{$\begin{array}{l}\text { Madhya } \\
\text { Pradesh }\end{array}$} & Balaghat & Baihar, Waraseoni, Lanji, Balaghat \\
\hline & Barwani & Sendhwa, Barwani, Pansemal \\
\hline & Betul & Betul, Bhainsdehi, Multai \\
\hline & Bhopal & Huzur, Berasia \\
\hline & Chhindwara & Amarwara, Chhindwara, Parasia, Sausar. \\
\hline & Damoh & Damoh \\
\hline & Dewas & Bagli, Dewas, Sonkatch \\
\hline & Dhar & Badnawar, Dhar, Kukshi, Manawar, Sardarpur \\
\hline & Dindori & Dindori \\
\hline & East Nimar & Burhanpur, Khandwa. \\
\hline & Harda & Harda \\
\hline & Hoshangabad & Sohagpur, Pipariya, Itarsi. \\
\hline & Indore & Depalpur, Mhow, Indore, Sawer \\
\hline & Jhabua & Jobat, Jhabua, Petlawad, Alirajpur, Petlawad, Thandla \\
\hline & Mandsaur & Mandsaur \\
\hline & Narsimhapur & Narsimhapur, Gadarwara \\
\hline & Raisen & Goharganj, Baraily, Udaipura, Raisen, Gairatganj, Silwani, Begamganj \\
\hline & Rajgarh & Sarangpur,Narsinghgarh,Rajgarh,Khilchipur,Biaora \\
\hline & Ratlam & Ratlam, Sailana, Jaora, A lot. \\
\hline & Rewa & Gurh,Mauganj,Sirmour,Teonthar \\
\hline & Sagar & Rehli, Sagar, Banda1. \\
\hline & Sehore & Nasrullaganj,Budni,Ashta,Ichhawar, Sehore \\
\hline & Satna & Maihar,Amarpatan,Nagod, Raghurajnagar \\
\hline & Seoni & Seoni, Lakhnadon. \\
\hline & Shahdol & Pushparajgarh,Anuppur,Sohagpur1,Jaisinghnagar,Beohari \\
\hline & Shajapur & Shajapur,Shujalpur,Agar,Susner \\
\hline & Sheopur & Sheopur,Vijaypur \\
\hline & Shivpuri & Kolaras,Khaniyadhana,Pohari,Shivpuri,Karera \\
\hline & Sidhi & Gopadbanas,Singrauli,Deosar \\
\hline & Tikamgarh & Tikamgarh,Jatara, Niwari \\
\hline & Ujjain & Badnagar, Khacharod, Ujjain, Tarana, Mahidpur. \\
\hline & Umaria & Bandhogarh \\
\hline & Vidisha & Vidisha,Basoda,Lateri,Kurwai,Sironj \\
\hline & West Nimar & Bhagwanpura,Jhiranya,Segaon,Khargone,Bhikangaon,Kasrawad,Barwaha,Ma \\
\hline
\end{tabular}




\begin{tabular}{|c|c|c|}
\hline & & heshwar \\
\hline \multirow[t]{37}{*}{ Bihar } & Araria & Araria \\
\hline & Banka & Banka \\
\hline & Begusarai & Begusarai \\
\hline & Bhagalpur & Bhagalpur, Naugachhia \\
\hline & Bhojpur & Arrah \\
\hline & Buxar & Buxar \\
\hline & Darbhanga & Benipur, Darbhanga. \\
\hline & Gaya & Gaya \\
\hline & Gopalganj & Gopalganj \\
\hline & Jamui & Jamui \\
\hline & Jehanabad & Jehanabad \\
\hline & Katihar & Katihar \\
\hline & Kaimur (Bhabua) & Bhabua \\
\hline & Khagaria & Khagaria \\
\hline & Katihar & Katihar \\
\hline & Khagaria & Khagaria \\
\hline & Kishanganj & Kishanganj \\
\hline & Lakhisarai & Lakhisarai \\
\hline & Madhepura & Madhepura, Udakishanganj \\
\hline & Madhubani & Jhanjharpur,Madhubani,Benipatti \\
\hline & Munger & Munger \\
\hline & Muzaffarpur & Purba muzaffarpur \\
\hline & Nalanda & Bihar, Hilsa. \\
\hline & Nawada & Nawada \\
\hline & Pashchim Champaran & Bettiah \\
\hline & Patna & Dinapur-Cum-Khagaul,Masaurhi,Patna city,Barh,Patna Rural \\
\hline & Purba Champaran & Motihari,Dhaka \\
\hline & Purnia & Purnia East \\
\hline & Rohtas & Sasaram \\
\hline & Saharsa & Sonbarsa \\
\hline & Samastipur & Rosera, Samastipur, Dalsinghsarai. \\
\hline & Saran & Chapra \\
\hline & Sheikhpura & Sheikhpura \\
\hline & Sheohar & Sheohar \\
\hline & Sitamarhi & Purba sitamarhi \\
\hline & Siwan & Siwan \\
\hline & Supaul & Supaul, Saraigarh Bhaptiyahi. \\
\hline
\end{tabular}




\begin{tabular}{|c|c|c|}
\hline & Vaishali & Hajipur \\
\hline \multirow[t]{10}{*}{ Gujarat } & Amreli & Jafrabad, Dhari, Rajula, Savar Kundla, Kunkavav Vadia, Amreli, Lilia, Babra. \\
\hline & Bhavnagar & Mahuva, Talaja, Palitana. \\
\hline & Dohad & Dohad, Limkheda, Jhalod. \\
\hline & Jamnagar & Kalyanpur, Bhanvad, Jamjodhpur, Khambhalia, Lalpur, Kalavad. \\
\hline & Junagadh & $\begin{array}{l}\text { Patan-Veraval, Kodinar, Una, Malia, Talala, Manavadar, Mangrol, Keshod, } \\
\text { Mendarda, Visavadar, Vanthali, Junagadh, Bhesan. }\end{array}$ \\
\hline & Narmada & Dediapada \\
\hline & Porbandar & Kutiyana, Ranavav, Porbandar. \\
\hline & Rajkot & Upleta, Dhoraji, Jetpur, Jamkandorna, Gondal. \\
\hline & The Dangs & The Dangs \\
\hline & Vadodara & Chhota Udaipur \\
\hline \multirow[t]{17}{*}{ West Bengal } & Bankura & Vishnupur, Bankura \\
\hline & Barddhaman & Burdwan - I, Kalna - I, Katoya. \\
\hline & Birbhum & Bolpur Sriniketan, Rampurhat, Suri - I. \\
\hline & Dakshin Dinajpur & Balurghat \\
\hline & Darjiling & Darjeeling Pulbazar \\
\hline & Haora & Shyampur-I, Sankrail, Uluberia-I \\
\hline & Hugli & Arambag, Serampur Uttarpara, Hugli. \\
\hline & Jalpaiguri & Jalpaiguri, Alipurduar \\
\hline & Koch Bihar & Dinhata - I, Tufanganj-I, Mathabhanga - II, Cooch Behar - I, Mekliganj, \\
\hline & Maldah & Maldah (Old) \\
\hline & Medinipur & Contai - I, Tamluk, Midnapore, Jhargram, Ghatal. \\
\hline & Murshidabad & Murshidabad Jiaganj, Berhampore, Jalangi, Kandi1. \\
\hline & Nadia & Kalyani, Ranaghat, Krishnagar - II. \\
\hline & $\begin{array}{l}\text { North Twenty Four } \\
\text { Paraganas }\end{array}$ & Basirhat - I, Bongaon \\
\hline & Puruliya & Puruliya-1 \\
\hline & $\begin{array}{l}\text { South Twenty Four } \\
\text { Paraganas }\end{array}$ & Diamond Harbour - I, Bhangar-I. \\
\hline & Uttar Dinajpur & Raiganj, Islampur. \\
\hline \multirow[t]{6}{*}{ Jharkhand } & Bokaro & Bermo \\
\hline & Chatra & Chatra \\
\hline & Deoghar & Deoghar \\
\hline & Dhanbad & Baghmara-Cum-Katras, Dhanbad-Cum-Kenduadih-Jagta. \\
\hline & Dumka & Dumka, Jamtara. \\
\hline & Giridih & Giridih \\
\hline
\end{tabular}




\begin{tabular}{|c|c|c|}
\hline & Godda & Godda \\
\hline & Gumla & Simdega, Gumla. \\
\hline & Hazaribagh & Hazaribag \\
\hline & Kodarma & Kodarma \\
\hline & Pakaur & Pakaur \\
\hline & Palamu & Latehar, Daltonganj. \\
\hline & $\begin{array}{l}\text { Pashchimi } \\
\text { Singhbhum }\end{array}$ & Chaibasa, Kharsawan, Chakradharpur. \\
\hline & Purbi Singhbhum & Dhalbhumgarh \\
\hline & Ranchi & Khunti, Ranchi. \\
\hline & Sahibganj & Rajmahal \\
\hline Uttar Pradesh & Agra & Kheragarh,Bah,Kiraoli,Agra,Etmadpur \\
\hline & Aligarh & Khair,Atrauli \\
\hline & Allahabad & Meja,Karchhana,Allahabad,Phulpur1,Handia, Soraon \\
\hline & Ambedkar Nagar & Akbarpur1,Tanda \\
\hline & Auraiya & Auraiya,Bidhuna \\
\hline & Azamgarh & Lalganj,Azamgarh,Phulpur, Sagri \\
\hline & Baghpat & Baghpat \\
\hline & Bahraich & Kaiserganj,Nanpara \\
\hline & Ballia & Ballia,Rasra,Bansdih \\
\hline & Balrampur & Utraula,Balrampur \\
\hline & Banda & Naraini,Banda,Baberu \\
\hline & Barabanki & Haidergarh,Nawabganj,Ramsanehighat \\
\hline & Bareilly & Aonla,Bareilly,Faridpur,Baheri \\
\hline & Basti & Basti,Harraiya \\
\hline & Bijnor & Bijnor,Dhampur,Nagina,Najibabad \\
\hline & Budaun & Dataganj,Budaun,Gunnaur,Sahaswan,Bisauli \\
\hline & Bulandshahar & Khurja,Bulandshahr,Anupshahr,Sikandrabad \\
\hline & Chandauli & Chakia,Chandauli,Sakaldiha \\
\hline & Chitrakoot & Karwi,Mau \\
\hline & Deoria & Salempur,Deoria \\
\hline & Etah & Jalesar,Etah,Aliganj,Kasganj \\
\hline & Etawah & Bharthana,Etawah \\
\hline & Faizabad & Bikapur,Faizabad \\
\hline & Farrukhabad & Kaimganj \\
\hline & Fatehpur & Khaga,Fatehpur,Bindki \\
\hline & Firozabad & Shikohabad,Firozabad,Jasrana \\
\hline & $\begin{array}{l}\text { Gautam } \quad \text { Buddha } \\
\text { Nagar }\end{array}$ & Dadri \\
\hline
\end{tabular}




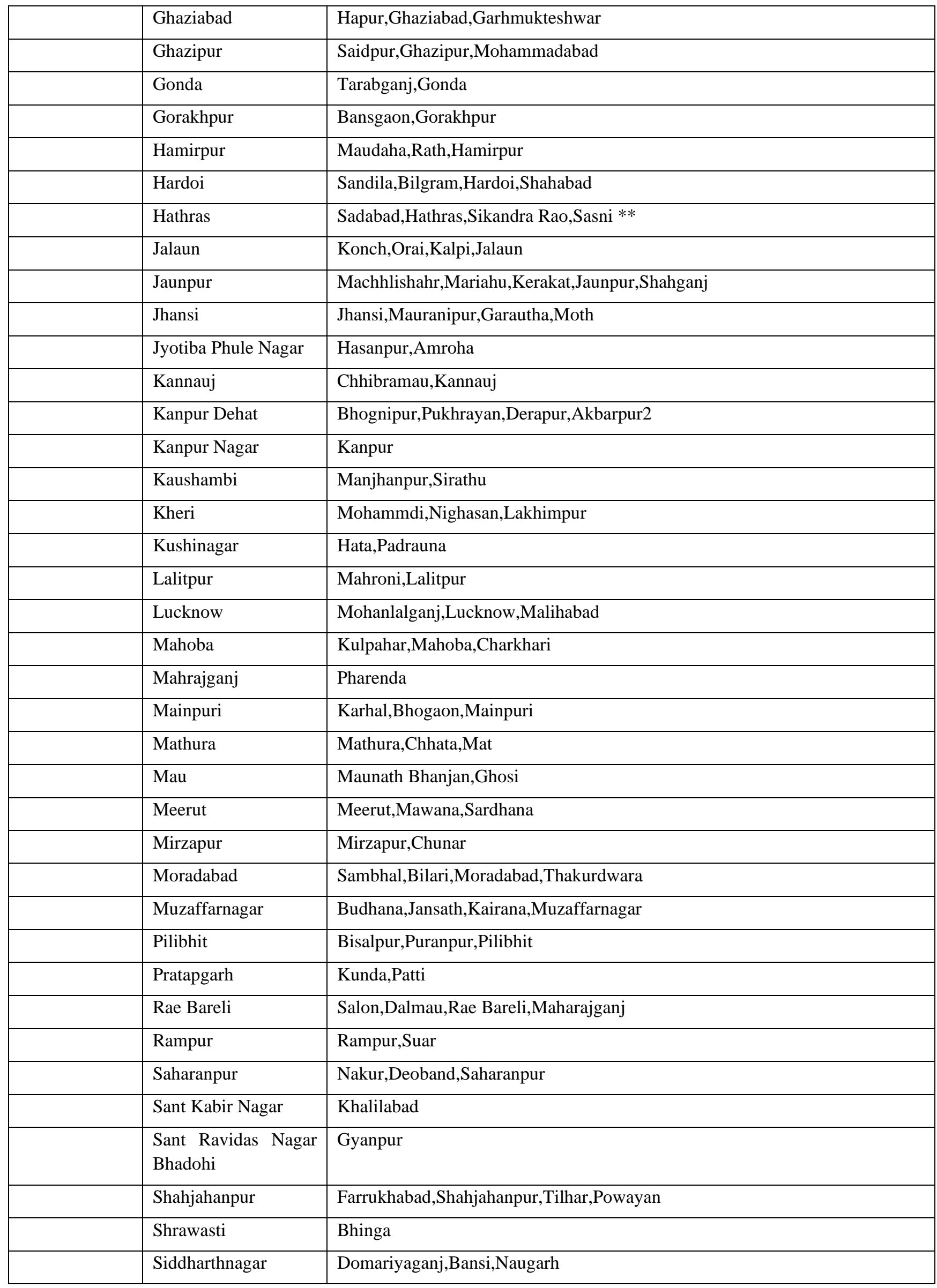




\begin{tabular}{|c|c|c|}
\hline & Sitapur & Misrikh,Sidhauli,Biswan \\
\hline & Sonbhadra & Dudhi,Robertsganj \\
\hline & Sultanpur & Amethi,Sultanpur,Kadipur,Gauriganj,Musafirkhana \\
\hline & Unnao & Purwa,Unnao,Safipur,Hasanganj \\
\hline & Varanasi & Varanasi \\
\hline \multirow[t]{30}{*}{ Rajasthan } & Ajmer & Beawar,Kekri,Bhinay,Sarwar,Ajmer,Nasirabad,Kishangarh \\
\hline & Alwar & $\begin{array}{l}\text { Thanagazi,Rajgarh1,Lachhmangarh,Alwar,Bansur,Ramgarh,Mandawar,Kisha } \\
\text { ngarh Bas,Behror,Tijara }\end{array}$ \\
\hline & Banswara & Kushalgarh,Kalinjara,Banswara,Garhi \\
\hline & Baran & Chhipabarod,Chhabra,Atru,Baran,Kishanganj,Shahbad \\
\hline & Barmer & Chohtan,Gudha Malani,Siwana,Sheo,Barmer,Pachpadra \\
\hline & Bharatpur & Bayana,Weir,Rupbas,Nadbai,Nagar,Deeg,Kaman \\
\hline & Bhilwara & $\begin{array}{l}\text { Sahara,Mandalgarh,Bhilwara,Kotri,Mandal,Jahazpur,Asind,BaneraShahpura, } \\
\text { Hurda }\end{array}$ \\
\hline & Bikaner & Kolayat,Nokha,Bikaner,Lunkaransar \\
\hline & Bundi & Bundi,Hindoli,Keshoraipatan,Nainwa \\
\hline & Chittaurgarh & $\begin{array}{l}\text { Arnod,Pratapgarh,BariSadri,Chhoti,Sadri,Nimbahera,Bhadesar,Kapasan, } \\
\text { Begun,Rashmi,Gangrar }\end{array}$ \\
\hline & Churu & Sujangarh,Dungargarh,Ratangarh,Churu,Sardarshahar,Taranagar \\
\hline & Dausa & Lalsot,Dausa,Sikrai,Mahwa,Baswa \\
\hline & Dhaulpur & Baseri,Bari,Dhaulpur \\
\hline & Dungarpur & Sagwara,Simalwara,Dungarpur,Aspur \\
\hline & Ganganagar & $\begin{array}{l}\text { Gharsana,Anupgarh,Vijainagar,Suratgarh,Raisinghnagar,Padampur, } \\
\text { Karanpur,Ganganagar,Sadulshahar }\end{array}$ \\
\hline & Hanumangarh & Rawatsar,Nohar,Bhadra,Pilibanga,Tibi,Hanumangarh,Sangaria \\
\hline & Jaipur & $\begin{array}{l}\text { Dudu (Hq. } \quad \text { Mauzamabad),Phagi,Chaksu,Sanganer,Bassi, } \\
\text { (Hq.Sambhar),Jaipur,Jamwa Ramgarh,Amber,Chomu,Viratnagar,Kotputli }\end{array}$ \\
\hline & Jaisalmer & Jaisalmer,Pokaran \\
\hline & Jalor & Sanchore,Raniwara,Bhinmal,Jalor,Ahore \\
\hline & Jhalawar & Gangdhar,Pirawa,Jhalrapatan,Aklera,Pachpahar,Khanpur \\
\hline & Jhunjhunun & Nawalgarh,Udaipurwati,Khetri,Jhunjhunun,Chirawa \\
\hline & Jodhpur & Jodhpur,Shergarh,Bilara,Bhopalgarh,Osian,Phalodi \\
\hline & Karauli & Sapotra,Karauli,Nadoti,Todabhim,Hindaun \\
\hline & Kota & Ramganj Mandi,Sangod,Ladpura,Digod,Pipalda \\
\hline & Nagaur & Merta,Degana,Parbatsar,Nagaur,Jayal,Nawa,Didwana,Ladnu \\
\hline & Pali & Bali,Desuri,Pali,Jaitaran,Sojat \\
\hline & Rajsamand & Nathdwara,Railmagra,Kumbhalgarh,Rajsamand,Amet, Deogarh,Bhim \\
\hline & Sawai Madhopur & Sawai Madhopur,Khandar,Bonli,Bamanwas \\
\hline & Sikar & Sikar,Sri Madhopur,Neem-Ka-Thana \\
\hline & Sirohi & Abu Road,Reodar,Pindwara,Sirohi,Sheoganj \\
\hline
\end{tabular}




\begin{tabular}{|l|l|l|}
\hline & Tonk & Uniara,Todaraisingh,Tonk,Malpura,Niwai \\
\hline & Udaipur & Kherwara,Phalsiya,Sarada,Salumbar,Kotra,Girwa,Vallabhnagar, Gogunda \\
\hline
\end{tabular}

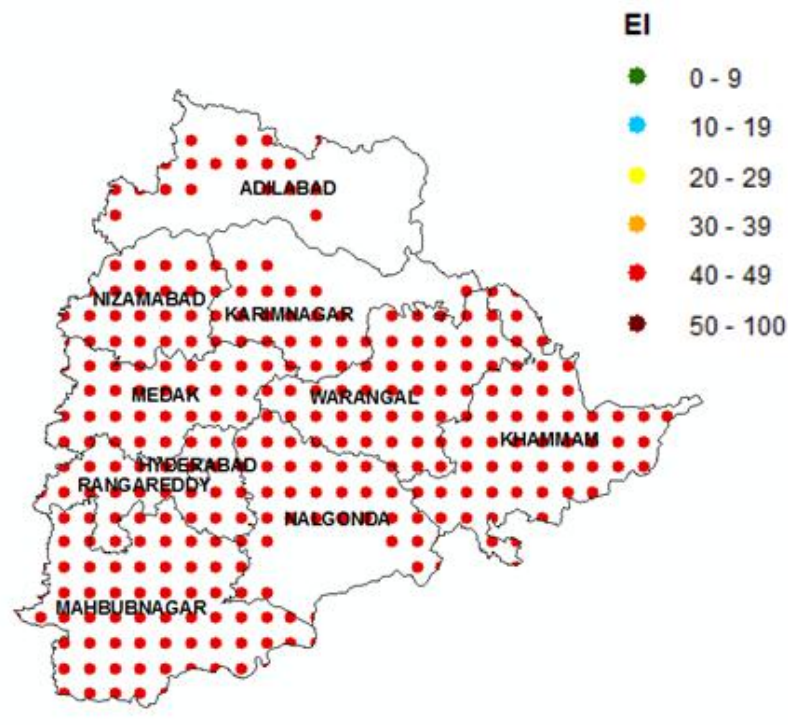

Fig 5a: Telangana

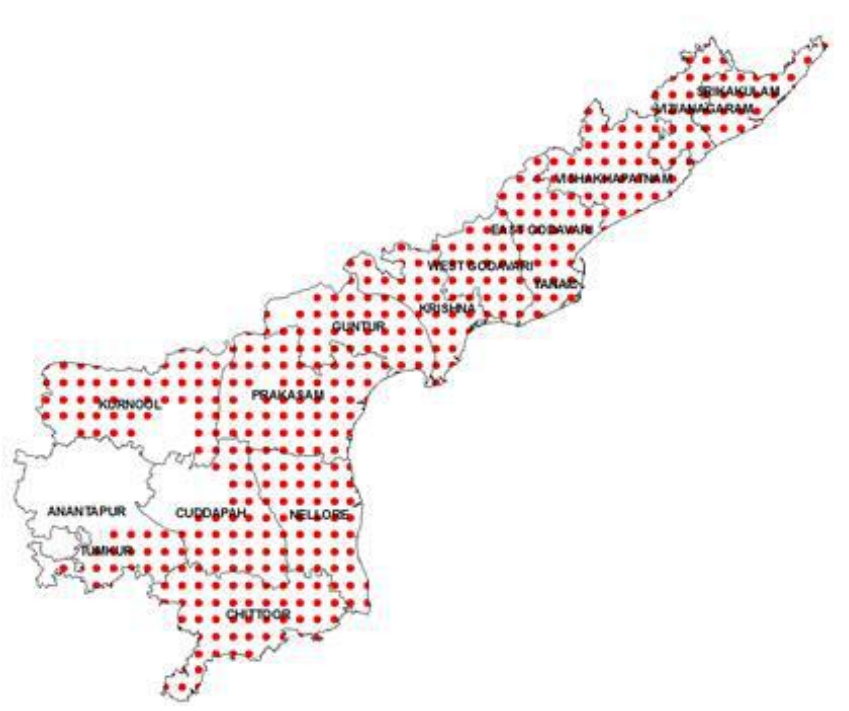

Fig 5b: Andhra Pradesh

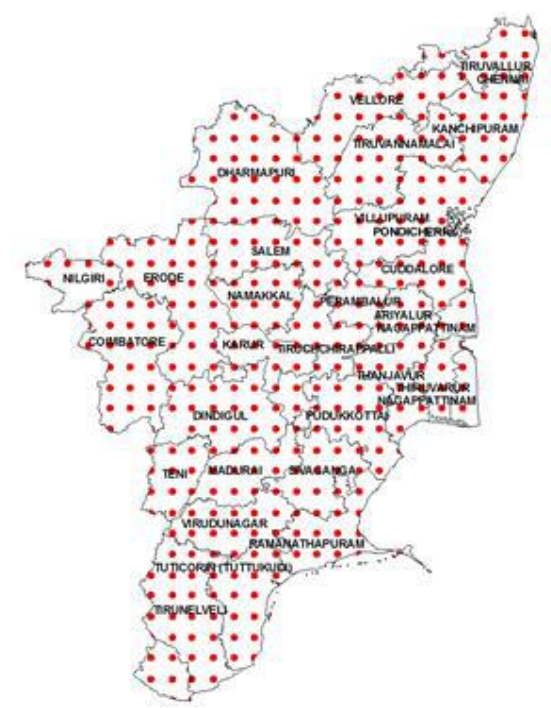

Fig 5c: Tamil Nadu

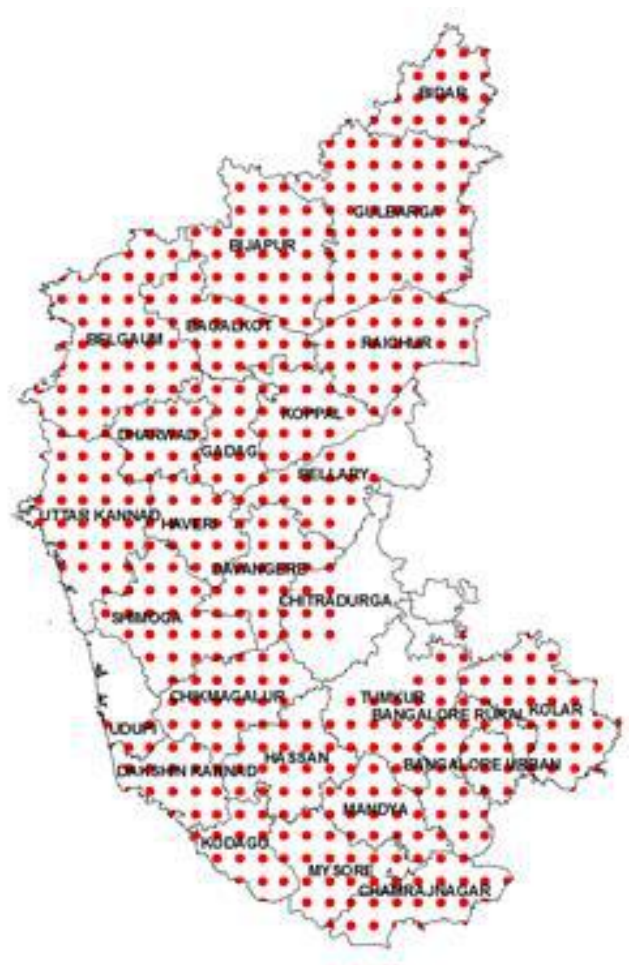

Fig 5d: Karnataka 

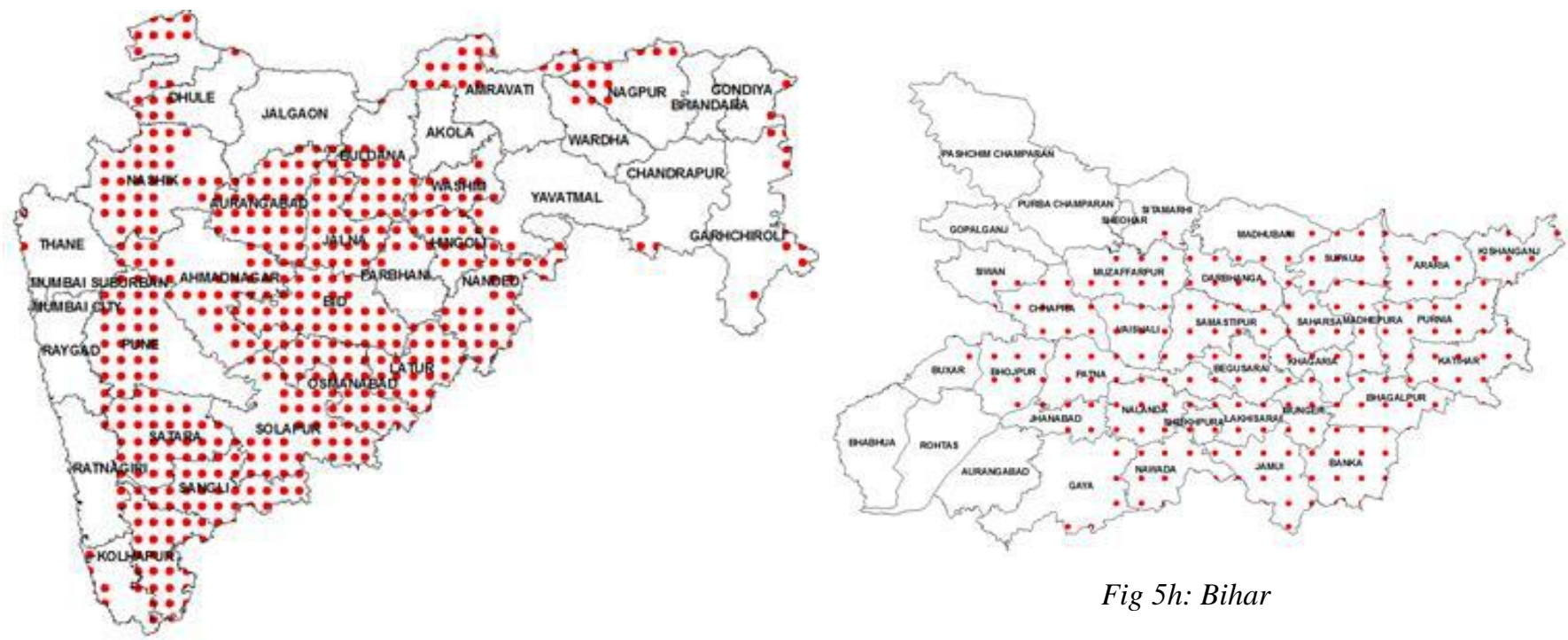

Fig 5h: Bihar

Fig 5e: Maharashtra

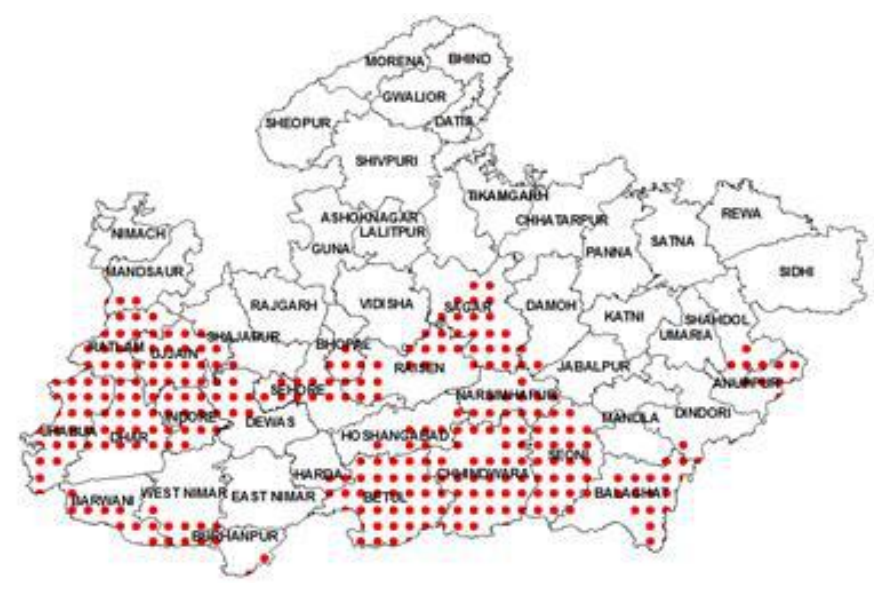

Fig 5f: Madhya Pradesh

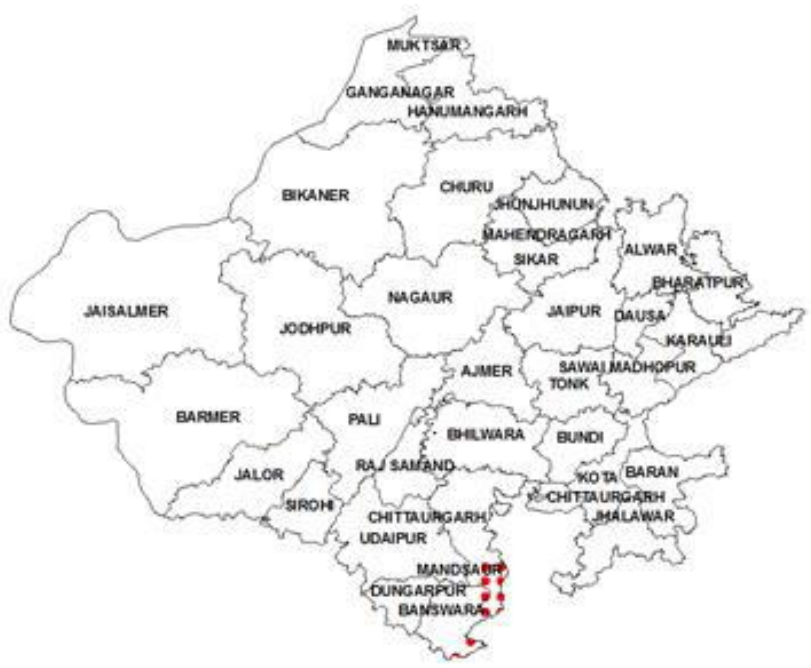

Fig 5i: Rajasthan
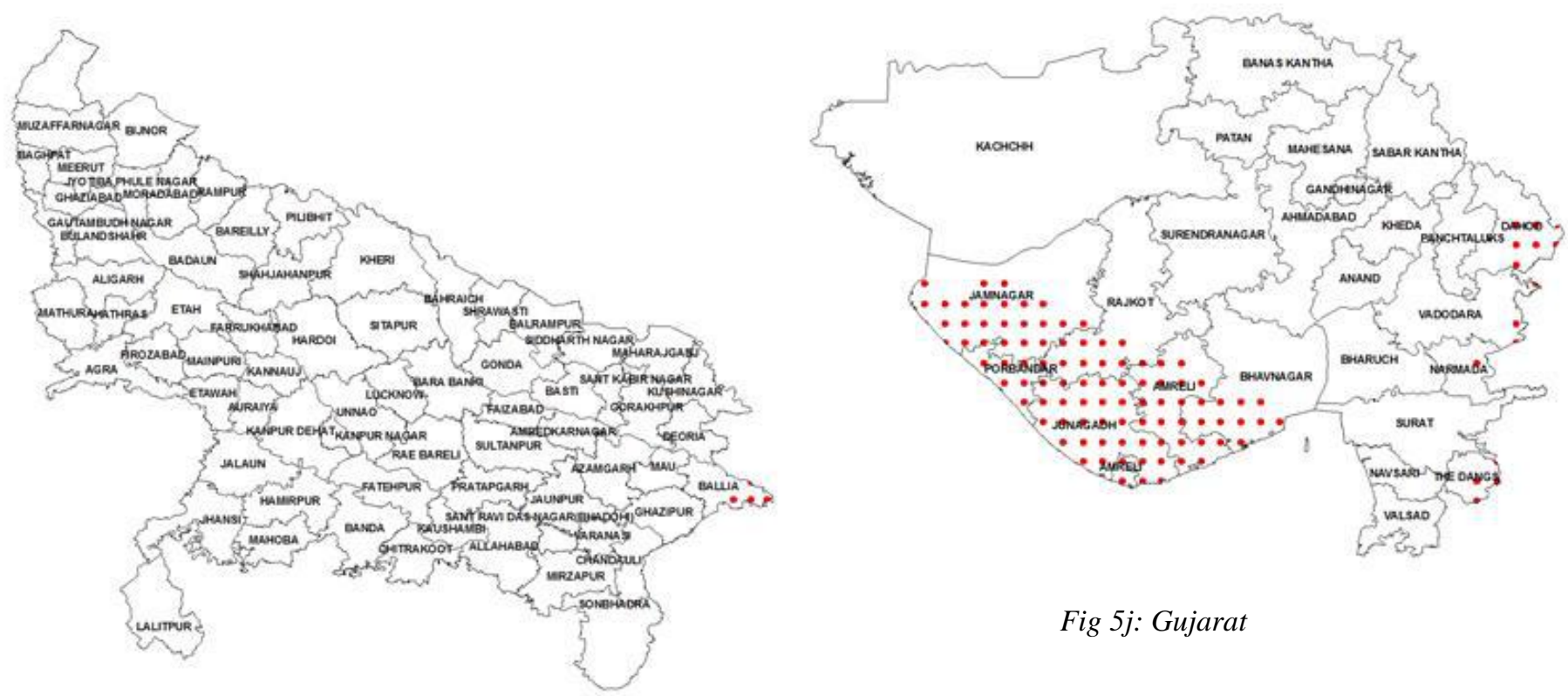

Fig 5j: Gujarat

Fig 5g: Uttar Pradesh 


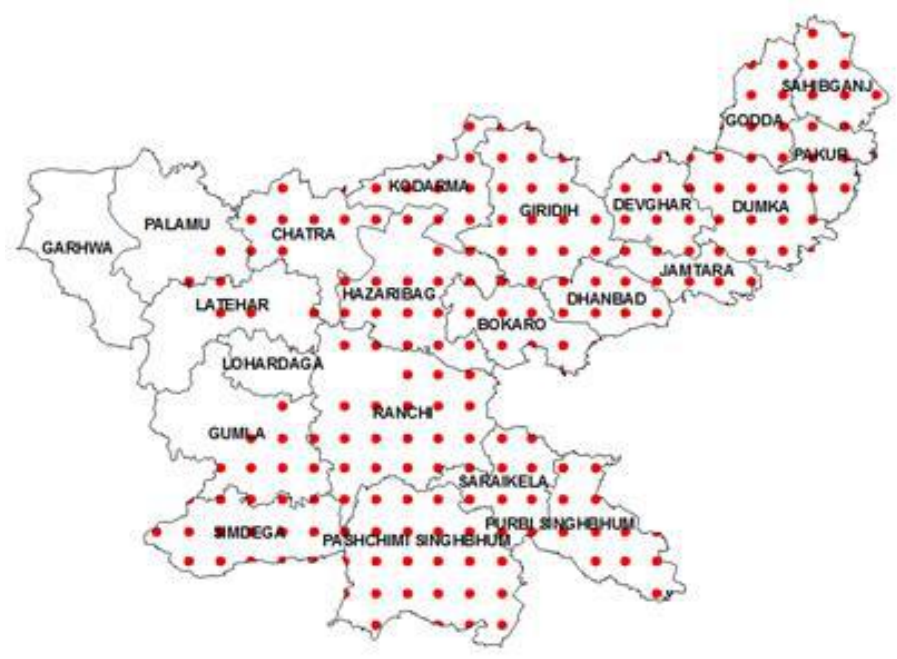

Fig 5l: Jharkhand

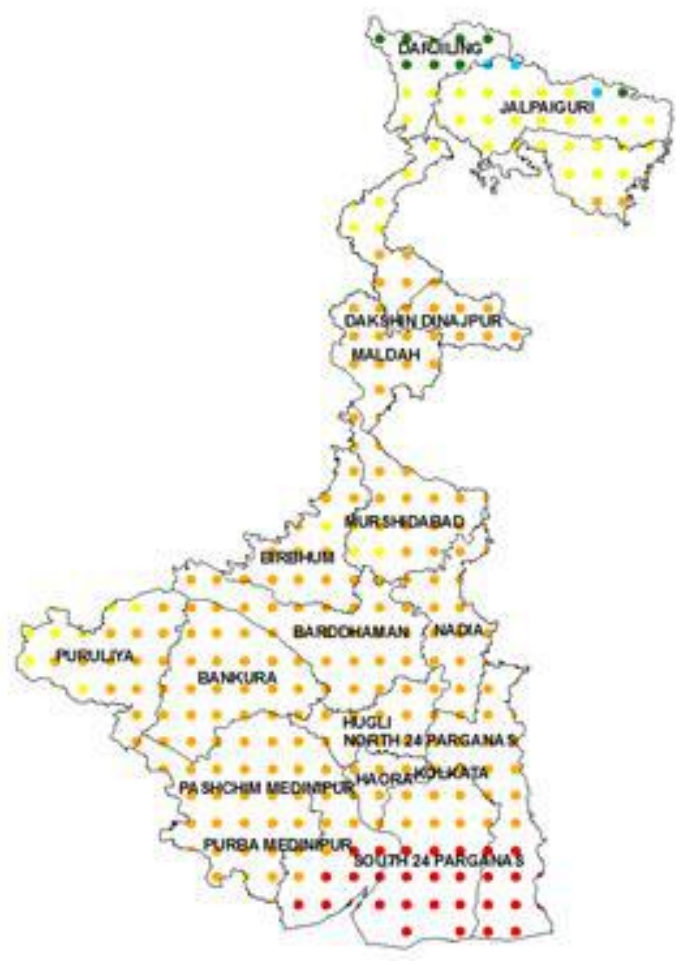

Fig 5k: West Bengal

Fig 5: Risk probability areas in the Major Maize Growing States

\section{ACKNOWLEDGEMENTS}

The authors are thankful to Smt. G. Jayalakshmi I.A.S.. Director General, NIPHM for her constant support and encouragement for conducting these studies. Further, the authors duly acknowledge the support provided by Dr. C. S. Murthy, Scientist G \& Head, Agriculture Science \& Applications and Dr. Abhishek Chakraborty, Scientsit/Engineer- SFA, National Remote Sensing Centre,
Hyderabad. A special thanks to Dr. V. Sridhar, Scientist, IIHR for his good guidance.

\section{REFERENCES}

4] Anon., 1997. Insect Control Guide, Ohio, USA: Meister Publishing Co.442 pp.

[5] Ashley TR, Wiseman BR, Davis FM, Andrews KL, 1989. The fall armyworm: a bibliography. Florida Entomologist, 72(1):152-202

[6] Bateman, M. L., Day, R. K., Luke, B., Edgington, S., Kuhlmann, U., Cock, M. J. W., 2018. Assessment of potential biopesticide options for managing fall armyworm (Spodoptera frugiperda) in Africa. Journal of Applied Entomology, 142(9), 805-819. https://onlinelibrary.wiley.com/journal/14390418 doi: $10.1111 /$ jen. 12565

[7] Baudron, F., Zaman-Allah, M. A., Chaipa, I., Chari, N., Chinwada, P. , 2019. Understanding the factors conditioning fall armyworm (Spodoptera frugiperda J.E. Smith) infestation in African smallholder maize fields and quantifying its impact on yield: a case study in Eastern Zimbabwe. Crop Protection, 120, 141-150.

[8] Behle, R. W., Popham, H. J. R., 2012. Laboratory and field evaluations of the efficacy of a fast-killing baculovirus isolate from Spodoptera frugiperda. Journal of Invertebrate Pathology,109(2), 194-200. http://www.sciencedirect.com/science/article/pii/S002220 $\underline{1111002400}$ doi: 10.1016/j.jip.2011.11.002

[9] Birhanu Sisay, Simiyu, J., Malusi, P., Likhayo, P., Esayas Mendesil, Elibariki, N., Mulatu Wakgari, Gashawbeza Ayalew, Tadele Tefera, 2018. First report of the fall armyworm, Spodoptera frugiperda (Lepidoptera: Noctuidae), natural enemies from Africa. Journal of Applied Entomology, 142(8), 800-804. https://onlinelibrary.wiley.com/journal/14390418

[10] Brown ES, Dewhurst CF, 1975. The genus Spodoptera (Lepidoptera, Noctuidae) in Africa and the Near East. Bulletin of Entomological Research, 65(2):221-262

[11] Busato, G. R., Grützmacher, A. D., Garcia, M. S., Giolo, F. P., Zotti, M. J., Bandeira, J. M., 2005. (Exigências 
térmicas e estimativa do número de gerações dos biótipos "milho" e "arroz" de Spodoptera frugiperda). Pesquisa Agropecuária Brasileira, 40(4), 329-335. https://dx.doi.org/10.1590/S0100-204X2005000400003

[12] CABI/EPPO, 1998. Distribution maps of quarantine pests for Europe (edited by Smith IM, Charles LMF). Wallingford, UK: CAB International, xviii $+768 \mathrm{pp}$

[13] Camera C, Dequech STB, Ribeiro Ldo P, Querino RB, 2010. First report of Trichogramma rojasi parasitizing eggs of Spodoptera frugiperda. (First report of Trichogramma rojasi parasitizing eggs of Spodoptera frugiperda.) Ciência Rural, 40(8):1828-1830. http://www.scielo.br/scielo.php?script=sci_arttext\&pid=S 0103-84782010000800025\&lng=en\&nrm=iso\&tlng=pt

[14] Casmuz, A., Juárez, M. L., Socías, M. G., Murúa, M. G., Prieto, S., Medina, S., Willink, E., Gastaminza, G., 2010. Revisión de los hospederos del gusano cogollero del maíz, Spodoptera frugiperda (Lepidoptera: Noctuidae). Revista de la Socie- dad Entomológica Argentina, 69, 209-231.

[15] Chimweta, M., Nyakudya, I. W., Jimu, L., Mashingaidze, A. B., 2019. Fall armyworm [Spodoptera frugiperda (J.E. Smith)] damage in maize: management options for floodrecession cropping smallholder farmers. International Journal of Pest Management, https://doi.org/10.1080/09670874.2019.1577514

[16] Chinwada, P. , 2018. Fall Armyworm Prevalence Assessment in Madagascar. In: FAO Mission Report . 32 pp.

[17] CIMMYT, 2018. Fall Armyworm in Africa: A Guide for Integrated Pest Management. First Edition, Prasanna, B. M., Huesing, J. E., Eddy, R., Peschke, V. M., eds. CIMMYT, Mexico, 109 pp.

[18] Cock, M. J. W., 1985. A review of biological control of pests in the Commonwealth Caribbean and Bermuda up to 1982, Farnham Royal, UK: Commonwealth Agricultural Bureaux. 218 pp.

[19] Cock, M. J. W., Beseh, P. K., Buddie, A. G., Cafá, G., Crozier, J., 2017. Molecular methods to detect Spodoptera frugiperda in Ghana, and implications for monitoring the spread of invasive species in developing countries. Scientific Reports, 7(1), 4103. doi: 10.1038/s41598-017-04238-y

[20] Cortez-Mondaca E, Armenta-Cárdenas I, Bahena-Juárez F, 2010. Parasitoids and percent parasitism of the fall armyworm (Lepidoptera: Noctuidae) in southern Sonora, Mexico. (Parasitoides y porcentaje de parasitismo sobre el Gusano Cogollero (Lepidoptera: Noctuidae) en el sur de Sonora, México.) Southwestern Entomologist, 35(2):199-203. http://sswe.tamu.edu/

[21] Crumb SE, 1956. The Larvae of the Phalaenidae. Technical Bulletin No. 1135. Washington DC, USA: United States Department of Agriculture

[22] Davis FM, Baker GT, Williams WP, 1995. Anatomical characteristics of maize resistant to leaf feeding by southwestern corn borer (Lepidoptera: Pyralidae) and fall armyworm (Lepidoptera: Noctuidae). Journal of Agricultural Entomology, 12(1):55-65
[23] Didonet J, Didonet APP, Erasmo EL, Santos GRdos, 2001. Incidence and population dynamics of pests and their natural enemies in upland rice in Gurupi, Tocantins. (Incidência e densidade populacional de pragas e inimigos naturais em arroz de terras altas, em GurupiTO.) Bioscience Journal, 17(1):67-76

[24] Early, R., González-Moreno, P., Murphy, S. T., Day, R., 2018. Forecasting the global extent of invasion of the cereal pest Spodoptera frugiperda, the fall armyworm. NeoBiota, (No.40), 25-50. https://neobiota.pensoft.net/article/28165/ doi: 10.3897/neobiota.40.28165

[25] EPPO, 2014. PQR database. Paris, France: European and Mediterranean Plant Protection Organization. http://www.eppo.int/DATABASES/pqr/pqr.htm

[26] EPPO, 2018. EPPO Global Database. EPPO Global Database, https://gd.eppo.int/ EPPO, Paris, France

[27] EPPO, 2019. Spodoptera frugiperda continues to spread in Asia. In: EPPO Reporting Service, (No. 2019/053) . Paris, France: EPPO.https://gd.eppo.int/reporting/article6483

[28] Estruch JJ, Warren GW, Mullins MA, Nye GJ, Craig JA, Koziel MG, 1996. Vip3A, a novel Bacillus thuringiensis vegetative insecticidal protein with a wide spectrum of activities against lepidopteran insects. Proceedings of the National Academy of Sciences of the United States of America, 93(11):5389-5394; 23 refs

[29] FAO, 2017a. FAO Advisory Note on Fall Armyworm (FAW) in Africa. In: FAO Advisory Note on Fall Armyworm (FAW) in Africa 7 pp. 5 June 2017. FAO, Rome, Italy

[30] FAO, 2017b. Briefing Note on FAP Actions on Fall Armyworm in Africa 15 December 2017. Briefing Note on FAP Actions on Fall Armyworm in Africa 15 December 2017, $\quad 7 \quad$ pp. http://www.fao.org/fileadmin/templates/fcc/map/map_of_ affected_areas/Fall_Armyworm_brief_-

_15Dec2017_.pdf FAO, Rome, Italy

[31] FAO, 2018. Briefing Note on FAO Actions on Fall Armyworm in Africa 31 January 2018. In: Briefing Note on FAO Actions on Fall Armyworm in Africa 31 January 2018 FAO, Rome, Italy, 6 pp

[32] FAO, 2018b. Briefing note on fall armyworm (FAW) in Africa. 16 February 2018, 7 pp. http://www.fao.org/3/abt415e.pdf

[33] FAO, 2018c. FAW Monitoring \& Early Warning System (FAMEWS). Rome, Italy: FAO.https://app.powerbi.com/view?r=eyJrIjoiMmFlOW QxMjctZjIwYy00MTdlLWJmMDgtMGM1ZWQ5YmZ mNDQwIiwidCI6IjJmMDYwNjMyLTg4MDgtNGM5ZS 05M2NmLTNmY2JkMWM1YTUxYiIsImMiOjh9\&refre $\mathrm{sh}=1$ \&pageName=ReportSection0901c9217ada50684ad0

[34] FAO, 2019a. FAO Statement on Fall Armyworm in Sri Lanka. Rome, Italy: FAO. http://www.fao.org/srilanka/news/detailevents/en/c/1177796/ 
[35] FAO, 2019b. First Detection of Fall Armyworm in China. Rome, Italy: FAO.https://www.ippc.int/fr/news/firstdetection-of-fall-armyworm-in-china/

[36] FAO, 2019c. Briefing note on FAO actions on fall armyworm. Rome, Italy: FAO.6 pp. http://www.fao.org/3/a-bs183e.pdf

[37] FAO, 2019d. FAW Briefing Note July 2019. Rome, Italy: FAO.

[38] FAO, 2019e. Food Chain Crisis Early Warning Bulletin, Rome, Italy: FAO (32), http://www.fao.org/3/ca5487en/ca5487en.pdf

[39] Fatoretto, J. C., Michel, A. P., Silva Filho, M. C., Silva, N., 2017. Adaptive potential of fall armyworm (Lepidoptera: Noctuidae) limits Bt trait durability in Brazil. Journal of Integrated Pest Management, 8(1), 17. https://academic.oup.com/jipm doi: 10.1093/jipm/pmx011

[40] Ganiger, P. C., Yeshwanth, H. M., Muralimohan, K., Vinay, N., Kumar, A. R. V., Chandrashekara, K., 2018. Occurrence of the new invasive pest, fall armyworm, Spodoptera frugiperda (J.E. Smith) (Lepidoptera: Noctuidae), in the maize fields of Karnataka, India. Current Science, 115(4), 621-623.

[41] Goergen, G., Kumar, P. L., Sankung, S. B., Togola, A., Tamò, M., 2016. First report of outbreaks of the fall armyworm Spodoptera frugiperda (J E Smith) (Lepidoptera, Noctuidae), a new alien invasive pest in West and Central Africa. PLoS ONE, 11(10), e0165632. http://journals.plos.org/plosone/article?id=10.1371/journa 1.pone. 0165632 doi: 10.1371/journal.pone. 0165632

[42] Gómez, J., Guevara, J., Cuartas, P., Espinel, C., Villamizar, L., 2013. Microencapsulated Spodoptera frugiperda nucleopolyhedrovirus: insecticidal activity and effect on arthropod populations in maize. Biocontrol Science and Technology, 23(7), 829-846. http://www.tandfonline.com/loi/cbst20 doi: 10.1080/09583157.2013.802288

[43] Greathead DJ, Greathead AH, 1992. Biological control of insect pests by insect parasitoids and predators: the BIOCAT database. Biocontrol News and Information, 13(4):61N-68N

[44] Guo, J. F., Zhao, J. Z., He, K. L., Zhang, F., Wang, Z. Y., 2018. Potential invasion of the crop-devastating insect pest fall armyworm Spodoptera frugiperda to China. Plant Protection, 44(6), 1-10.

[45] Gurrola-Pérez, C. C., Álvarez-Zagoya, R., HernándezMendoza, J. L., Correa-Ramírez, M., Pérez-Santiago, G., 2018. Record of Lespesia archippivora, Lespesia postica, and Archytas marmoratus parasitizing larvae of Spodoptera frugiperda in Durango, Mexico. (Registro de Lespesia archippivora, Lespesia postica, y Archytas marmoratus Parasitando larvas de Spodoptera frugiperda en Durango, México). Southwestern Entomologist, 43(2), 505-510. http://www.bioone.org/loi/swen doi: 10.3958/059.043.0221

[46] Haase, S., Sciocco-Cap, A., Romanowski, V., 2015. Baculovirus insecticides in Latin America: historical overview, current status and future perspectives. Viruses,
7(5), 2230-2267. http://www.mdpi.com/19994915/7/5/2230/htm

[47] Hailu, G., Niassy, S., Khan Z. R., Ochatum, N., Subramanian, S., 2018. Maize-legume intercropping and Push-pull for management of fall armyworm, stemborers and striga in Uganda. Agronomy Journal, 110, 1-10. doi: 10.2134/agronj2018.02.0110

[48] Harrison, R. D., Thierfelder, C., Baudron, F., Chinwada, P., Midega, C., Schaffner, U., van den Berg, J., 2019. Agro-ecological options for fall armyworm (Spodoptera frugiperda JE Smith) management: Providing low-cost, smallholder friendly solutions to an invasive pest. Journal of Environmental Management, 243, 318-330. https://doi.org/10.1016/j.jenvman.2019.05.011

[49] Hay-Roe, M. M., Meagher, R. L., Nagoshi, R. N., Newman, Y., 2016. Distributional patterns of fall armyworm parasitoids in a corn field and a pasture field in Florida. Biological Control, 96, 48-56. http://www.sciencedirect.com/science/article/pii/S104996 4416300147 doi: 10.1016/j.biocontrol.2016.02.003

[50] HugoDe Groote, Simon C. Kimenju, Bernard Munyua, Sebastian Palmasa, Menale Kassiec, Anani Brucea, 2020. Spread and impact of fall armyworm (Spodoptera frugiperda J.E. Smith) in maize production areas of Kenya. Agriculture, Ecosystems \& Environment, Volume 292, 15 April 2020.

[51] Hruska AJ, Gould F, 1997. Fall armyworm (Lepidoptera: Noctuidae) and Diatraea lineolata (Lepidoptera: Pyralidae): impact of larval population level and temporal occurrence on maize yield in Nicaragua. Journal of Economic Entomology, 90(2):611-622; 27 ref

[52] Hruska, A. J., 2019. Fall armyworm (Spodoptera frugiperda) management by smallholders. CAB Reviews, 14(043), 1-11. http://www.cabi.org/cabreviews/review/20193352460 doi: 10.1079/PAVSNNR201914043

[53] Huis, A. van, 1981. Integrated pest management in the small farmer's maize crop in Nicaragua. Mededelingen Landbouwhogeschool Wageningen, 81(6):221 pp

[54] ICAR-NBAIR, 2018a. PEST ALERT: 30th July, 2018. Spodoptera frugiperda (Smith, J.E.) (Insecta: Lepidoptera).

http://www.nbair.res.in/recent_events/Pest\%20Alert\%20 30th\%20July\%202018-new1.pdf

[55] ICAR-NBAIR, 2018b. Spodoptera frugiperda (J. E. Smith). Insects in Indian Agrosystems. ICAR-National Bureau of Agricultural Insect Resources (NBAIR), India. http://www.nbair.res.in/insectpests/Spodoptera_frugiperd a.php

[56] IITA, 2016. First report of outbreaks of the "Fall Armyworm" on the African continent. IITA Bulletin, No. 2330. http://bulletin.iita.org/index.php/2016/06/18/firstreport-of-outbreaks-of-the-fall-armyworm-on-the-africancontinent/

[57] IITA, 2018: Fall armyworm has reached the Indian subcontinent! Ibadan, Nigeria: IITA. http://www.iita.org/news-item/fall-armyworm-hasreached-the-indian-subcontinent/ 
[58] IPPC, 2016. The damage caused by Spodoptera frugiperda. (Les dégâts causés par Spodoptera frugiperda). In: IPPC Official Pest Report. Rome, Italy: FAO.https://www.ippc.int/

[59] IPPC, 2017a. First detection of Fall Army Worm (Spodoptera frugiperda). In: IPPC Official Pest Report, (No. ZAF-33/1) . Rome, Italy: FAO.https://www.ippc.int/

[60] IPPC, 2017b. Detection of Fall Army Worm Spodoptera frugiperda in Swaziland. In: IPPC Official Pest Report, (No. SWZ-02/1) . Rome, Italy: FAO.https://www.ippc.int/

[61] IPPC, 2017c. Occurrence of Fall Arm Worm (Spodoptera frugiperda) in Mozambique. In: IPPC Official Pest Report, (No. MOZ-06/1) . Rome, Italy: FAO.https://www.ippc.int/

[62] IPPC, 2017d. Preliminary Report on Fall Armyworm in Zambia. In: IPPC Official Pest Report, (No. ZMB-02/2). Rome, Italy: FAO.https://www.ippc.int/

[63] IPPC, 2017e. First report of the fall army worm Spodoptera frugiperda in Cameroon. In: IPPC Official Pest Report , (No. CMR-04/6) . Rome, Italy: FAO.https://www.ippc.int/

[64] IPPC, 2017f. Rapport d'étape sur la chenille légionnaire d'automne. In: IPPC Official Pest Report, (No. BFA01/1) . Rome, Italy, FAO.https://www.ippc.int/

[65] IPPC, 2018. Report on Fall armyworm (Spodoptera frugiperda). In: IPPC Official Pest Report , (No. GHA01/4) . Rome, Italy: FAO.https://www.ippc.int/

[66] IPPC, 2018b. First detection of Fall Army Worm on the border of Thailand. IPPC Official Pest Report, No. THA03/1 . FAO: Rome, Italy. https://www.ippc.int/

[67] IPPC, 2019a. First Detection Report of the Fall Armyworm Spodoptera frugiperda (Lepidoptra: Noctuidae) on Maize in Myanmar. IPPC Official Pest Report, No. MMR-19/2. Rome, Italy: FAO. https://www.ippc.int/

[68] IPPC, 2019b. Report of first detection of Fall Armyworm(FAW) in Republic of Korea. IPPC Official Pest Report, No. KOR-08/2. FAO: Rome, Italy. https://www.ippc.int/

[69] IPPC, 2019c. Report of first detection of Spodoptera frugiperda - Fall Armyworm (FAW) in Egypt. IPPC Official Pest Report, No. EGY-01/1. FAO: Rome, Italy. https://www.ippc.int/

[70] IPPC, 2019d. Report of first detection of Spodoptera frugiperda - Fall Armyworm (FAW) in Japan. IPPC Official Pest Report, No. JPN-08/6. https://www.ippc.int/

[71] IPPC, 2019e. The Occurence of Fall Armyworm (Spodoptera frugiperda) in Indonesia. IPPC Official Pest Report, No. IDN-04/1. Rome, Italy: FAO. https://www.ippc.int/

[72] IPPC, 2019f. Spodoptera frugiperda (Fall Armyworm). IPPC Official Pest Report, No. NPL-04/3. https://www.ippc.int/

[73] IPPC, 2019g. (Signalement de la chenille légionnaire d'automne (CLA) au Gabon). In: IPPC Official Pest Report, (No. GAB-03/2) Rome, Italy: FAO.https://www.ippc.int/
[74] IPPC, 2019h. Reporting Fall Armyworm (Spodoptera frugiperda) in Yemen. In: IPPC Official Pest Report, (No. NRO-03/1) Rome, Italy: FAO.https://www.ippc.int/

[75] IPPC, 2019i. Report of first detection of Fall Army Worm (FAW) in the Republic of the Philippines. In: IPPC Official Pest Report, (No. PHL-02/1) . Rome, Italy: FAO.https://www.ippc.int/

[76] IPPC, 2019j. Fall Armyworm (Spodoptera frugiperda) Control. In: IPPC Official Pest Report, (No. MYS-02/2) Rome, Italy: FAO.https://www.ippc.int/

[77] IPPC, 2020. First detection of Spodoptera frugiperda (fall armyworm) in Torres Strait. Rome, Italy: FAO.https://www.ippc.int/

[78] Jacobs, A., van Vuuren, A., Rong, I. H., 2018. Characterisation of the fall armyworm (Spodoptera frugiperda J.E. Smith) (Lepidoptera: Noctuidae) from South Africa. African Entomology, 26(1), 45-49.

[79] Jeger, M., Bragard, C., Caffier, D., Candresse, T., Chatzivassiliou, E., Dehnen-Schmutz, K., Gilioli, G., Gregoire, J. C., Miret, J. A. J., Navarro, M. N., Niere, B., Parnell, S., Potting, R., Rafoss, T., Rossi, V., Urek, G., Bruggen, A. van, Werf, W. van D., West, J., Winter, S., Gardi, C., Aukhojee, M., MacLeod, A., 2017. Pest categorisation of Spodoptera frugiperda. EFSA Journal,15(7),e04927.

http://onlinelibrary.wiley.com/wol1/doi/10.2903/j.efsa.20 17.4927/full

[80] Jeger, M., Bragard, C., Caffier, D., Candresse, T., Chatzivassiliou, E., Dehnen-Schmutz, K., Gilioli, G., Grégoire, J. C., Miret, J. A. J., Navarro, M. N., Niere, B., Parnell, S., Potting, R., Rafoss, T., Rossi, V., Urek, G., Bruggen, A. van, Werf, W. van der, West, J., Winter, S., Day, R., Early, R., Hruska, A., Nagoshi, R., Gardi, C., Mosbach-Schultz, O. (et al), 2018. Pest risk assessment of Spodoptera frugiperda for the European Union. EFSA Journal, 16(8), e05351. https://efsa.onlinelibrary.wiley.com/doi/full/10.2903/j.efs a.2018.5351

[81] Johnson SJ, 1987. Migration and the life history strategy of the fall armyworm, Spodoptera frugiperda in the western hemisphere. Insect Science and its Application, 8(4-6):543-549

[82] Kenis, M., Plessis, H. du, Berg, J. van den, Ba, M. N., Goergen, G., Kwadjo, K. E., Baoua, I., Tadele Tefera, Buddie, A., Cafà, G., Offord, L., Rwomushana, I., Polaszek, A., 2019. Telenomus remus, a candidate parasitoid for the biological control of Spodoptera frugiperda in Africa, is already present on the continent. Insects, 10(4), 92. https://www.mdpi.com/20754450/10/4/92/htm doi: 10.3390/insects 10040092

[83] King ABS, Saunders JL, 1984. The invertebrate pests of annual food crops in Central America. A guide to their recognition and control. London, UK: Overseas Development Administration

[84] Klun JA, Potts WJE, Oliver JE, 1996. Four species of noctuid moths degrade sex pheromone by a common antennal metabolic pathway. Journal of Entomological Science, 31(4):404-413; 16 ref 
[85] Komivi, S. A., Kimemia, J. W., Ekesi, S., Khamis, F. M., Ombura, O. L., Subramanian, S. , 2019. Ovicidal effects of entomopathogenic fungal isolates on the invasive Fall armyworm Spodoptera frugiperda (Lepidoptera: Noctuidae). Journal of Applied Entomology, doi: $10.1111 /$ jen. 12634

[86] Levy R, Habeck DH, 1976. Descriptions of the larvae of Spodoptera sunia and S. latifascia with a key to the mature Spodoptera larvae of the eastern United States (Lepidoptera: Noctuidae). Annals of the Entomological Society of America, 69(4):585-588

[87] Lewter, J. A., Szalanski, A. L., Nagoshi, R. N., Meagher, R. L., Jr., Owens, C. B., Luttrell, R. G., 2006. Genetic variation within and between strains of the fall armyworm, Spodoptera frugiperda (Lepidoptera: Noctuidae). Florida Entomologist, 89(1), 63-68. http://www.fcla.edu/FlaEnt/fe89p63.pdf doi: 10.1653/0015-4040(2006)89[63:GVWABS]2.0.CO;2

[88] Lu YangJiang, Adang, M. J., 1996. Distinguishing fall armyworm (Lepidoptera: Noctuidae) strains using a diagnostic mitochondrial DNA marker. Florida Entomologist, 79(1), 48-55. doi: 10.2307/3495753

[89] Luginbill, P. , 1928. Technical Bulletin. United States Department of Agriculture, Washington, D.C (34), 91 pp.

[90] Martel P, Hudon M, Ritchot C, 1980. The incidence of insect pests in certain crops in the south-west of Quebec in 1979. Annals of the Entomological Society of Quebec, 25(3):190-194

[91] Meagher, R. L., Jr., Nuessly, G. S., Nagoshi, R. N., HayRoe, M. M., 2016. Parasitoids attacking fall armyworm (Lepidoptera: Noctuidae) in sweet corn habitats. Biological Control, 95, 66-72. http://www.sciencedirect.com/science/article/pii/S104996 4416300068 doi: 10.1016/j.biocontrol.2016.01.006

[92] Midega, C. A. O., Pittchar, J. O., Pickett, J. A., Hailu, G. W., Khan, Z. R., 2018. A climate-adapted push-pull system effectively controls fall armyworm, Spodoptera frugiperda (J E Smith), in maize in East Africa. Crop Protection, 105, 10-15. http://www.sciencedirect.com/science/journal/02612194 doi: 10.1016/j.cropro.2017.11.003

[93] Mihm JA, Smith ME, Deutsch JA, 1988. Development of open-pollinated varieties, non-conventional hybrids and inbred lines of tropical maize with resistance to fall armyworm, Spodoptera frugiperda (Lepidoptera: Noctuidae), at CIMMYT. Florida Entomologist, 71(3):262-268

[94] Ministerio de Agricultura y Ganadería Servicio Fitosanitario del Estado (Costa Rica), 2009. Listado de Plagas (insectos y ácaros) en cultivos de importancia económica en Costa Rica (C). http://www.protecnet.go.cr/laboratorios/plagcul/cultivoc. htm

[95] Ministry of Agriculture of the State of Eritrea, 2018. Eritrea: Crops are safe from Fall Army Worm infestation. Fall Army Worm Newsletter, No. 3, August 2018. https://www.ippc.int/static/media/files/countrynews/2018 /08/10/FAW_Newsletter_August.pdf
[96] Moar WJ, Pusztai-Carey M, Faassen Hvan, Bosch D, Frutos R, Rang C, Luo K, Adang MJ, 1995. Development of Bacillus thuringiensis CryIC resistance by Spodoptera exigua (Hübner) (Lepidoptera: Noctuidae). Applied and Environmental Microbiology, 61(6):2086-2092; 35 ref

[97] Molina-Ochoa, J., Carpenter, J. E., Heinrichs, E. A., Foster, J. E., 2003. Parasitoids and parasites of Spodoptera frugiperda (Lepidoptera: Noctuidae) in the Americas and Caribbean Basin: an inventory. Florida Entomologist, 86(3), 254-289. http://www.fcla.edu/FlaEnt/ doi: 10.1653/00154040(2003)086[0254:PAPOSF]2.0.CO;2

[98] Montezano, D. G., Specht, A., Sosa-Gómez, D. R., Roque-Specht, V. F., Sousa-Silva, J. C., Paula-Moraes, S. V., Peterson, J. A., Hunt, T. E., 2018. Host plants of Spodoptera frugiperda (Lepidoptera: Noctuidae) in the Americas. African Entomology, 26(2), 286-300.

[99] Naeem-Ullah, U., Ashraf Ansari, M., Iqbal, N., Saeed, S., 2019. First authentic report of Spodoptera frugiperda (J.E. Smith) (Noctuidae: Lepidoptera) an alien invasive species from Pakistan. Applied Sciences and Business Economics, 6(1), 1-3. http://www.asbejournal.org/wpcontent/uploads/2019/09/1_First_authentic_report_of_Sp odoptera_frugiperda_J.E.SmithNoctuidae_Lepidoptera_a n_alien_invasive_species_from_Pakistan_ASBE_v6_i1_ p1_2019.pdf

[100] Nagoshi, R. N., Fleischer, S., Meagher, R. L., Hay-Roe, M., Khan, A., Murúa, M. G., Silvie, P., Vergara, C., Westbrook, J., 2017. Fall armyworm migration across the Lesser Antilles and the potential for genetic exchanges between North and South American populations. PLoS ONE, 12(2), e0171743. http://journals.plos.org/plosone/article?id=10.1371/journa 1.pone.0171743 doi: 10.1371/journal.pone.0171743

[101] Nagoshi, R. N., Meagher, R. L., Hay-Roe, M., 2012. Inferring the annual migration patterns of fall armyworm (Lepidoptera: Noctuidae) in the United States from mitochondrial haplotypes. Ecology and Evolution, 2(7), 1458-1467.

http://onlinelibrary.wiley.com/doi/10.1002/ece3.268/full doi: 10.1002/ece3.268

[102] Nagoshi, R. N., Murúa, M. G., Hay-Roe, M., Juárez, M. L., Willink, E., Meagher, R. L., 2012. Genetic characterization of fall armyworm (Lepidoptera: Noctuidae) host strains in Argentina. Journal of Economic Entomology, 105(2), 418-428.

[103] Nagoshi, R. N., Silvie, P., Meagher, R. L., Lopez, J., Machado, V., 2007. Identification and comparison of fall armyworm (Lepidoptera: Noctuidae) host strains in Brazil, Texas, and Florida. Annals of the Entomological Society of America, 100(3), 394-402. http://docserver.ingentaconnect.com/deliver/connect/esa/ 00138746/v100n3/s8.pdf?expires $=1202119290 \&$ id $=0000$ \&titleid=10263\&checksum=73980337A2767955F452EA F3BAB88E5B doi: 10.1603/00138746(2007)100[394:IACOFA]2.0.CO;2

[104] Nagoshi,R. N., Goergen, G., Agbeko, K. T., Agboka, K., Koffi, D., Meagher, R. L., 2018. Analysis of strain 
distribution, migratory potential, and invasion history of fall armyworm populations in northern Sub-Saharan Africa. Scientific Reports, 8, 3710. doi: doi:10.1038/s41598-018-21954-1

[105] OEPP/EPPO, 2015. EPPO Standards PM 7/124(1) Diagnostic protocol for Spodoptera littoralis, Spodoptera litura, Spodoptera frugiperda, Spodoptera eridania. Bulletin OEPP/EPPO Bulletin, 34:257-270

[106] Omoto C, Bernardi O, Salmeron E, Sorgatto RJ, Dourado PM, Crivellari A, Carvalho RA, Willse A, Martinelli S, Head GP, 2016. Field-evolved resistance to Cry1Ab maize by Spodoptera frugiperda in Brazil. Pest Management $\quad$ Science, 72(9):1727-1736. http://onlinelibrary.wiley.com/doi/10.1002/ps.4201/abstra ct

[107] Otim, M. H., Tay, W. T., Walsh, T. K., Kanyesigye, D., Adumo, S., Abongosi, J., Ochen, S., Sserumaga, J., Alibu, S., Abalo, G., Asea, G., Agona, A., 2018. Detection of sister-species in invasive populations of the fall armyworm Spodoptera frugiperda (Lepidoptera: Noctuidae) from Uganda. PLoS ONE, 13(4), e0194571. http://journals.plos.org/plosone/article?id=10.1371/journa 1.pone.0194571 doi: 10.1371/journal.pone.0194571

[108] Pair, S. D., Sparks, A. N., 1986. Evidence of annual long distance migration by the fall armyworm. In: Long-range migration of moths of agronomic importance to the United States and Canada: Specific examples of occurrence and synoptic weather patterns conducive to migration (ESA Symposium, 1982). U.S. Department of Agriculture, Misc. Publ, (ARS-43) [ed. by Sparks, A. N.]. Washington DC, USA: USDA. 25-33.

[109] Partha Sarathi Biswas, 2018. First case of fall armyworm infestation in state reported from Solapur district. The Indian Express, 27 September, 2018.

[110] Pitre HN, 1985. Insect problems on sorghum in the USA. Proceedings of the international sorghum entomology workshop, 15-21 July 1984, Texas A \& M University, College Station, Texas, USA. Patancheru, Andhra Pradesh, India: International Crops Research Institute for the Semi-Arid Tropics, 73-81

[111] Pratissoli D, Polanczyk RA, Grecco ED, Ferreira RA, Holtz AM, 2007. Microbial toxicity of new Bacillus thuringiensis isolates in two populations of Spodoptera frugiperda originating from Minas Gerais and Espírito Santo. (Efeito entomotóxico de novos isolados de Bacillus thuringiensis em duas populações de Spodoptera frugiperda oriundas de Minas Gerais e do Espírito Santo.) Revista Brasileira de Milho e Sorgo, 6(2):140148

[112] Rambajan I, 1981. Major insect pests of paddy in Guyana. International Rice Research Newsletter, 6(6):1617

[113] Ramirez-Garcia L, Bravo Mojica H, Llanderal Cazares C, 1987. Development of Spodoptera frugiperda (J.E. Smith) (Lepidoptera: Noctuidae) under different conditions of temperature and humidity. Agrociencia, 67:161-171
[114] Rwomushana, I., Bateman, M., Beale, T., Beseh, P., Cameron, K., Chiluba, M., Clottey, V., Davis, T., Day, R., Early, R., Godwin, J., Gonzalez-Moreno, P., Kansiime, M., Kenis, M., Makale, F., Mugambi, I., Murphy, S., Nunda, W., Phiri, N., Pratt, C., Tambo, J., 2018. Fall armyworm: impacts and implications for Africa. In: Fall armyworm: impacts and implications for Africa . Wallingford, UK: CABI.51 pp. https://www.invasive-species.org/wpcontent/uploads/sites/2/2019/02/FAW-Evidence-NoteOctober-2018.pdf

[115] Sarfaraz Ali, Zakkia Masroor, Mohammad Danish Masroor, 2018. First record of the fall armyworm, Spodoptera frugiperda (J. E. Smith, 1797) (Lepidoptera: Noctuidae), an evil attack on paddy in Magadh, Bihar (India). International Journal of Emerging Technologies and Innovative Research (www.jetir.org), 5(12), 546-549. http://www.jetir.org/papers/JETIR1812380.pdf

[116] Seymour PR, Roberts H, Davis ME (Compilers), 1985. Insects and other invertebrates found in plant material imported into England and Wales, 1984. Reference Book, Ministry of Agriculture, Fisheries and Food, UK, 442/84

[117] Sharanabasappa, Kalleshwara swamy, C. M., 2018. Presence of fall armyworm, Spodoptera frugiperda (J E Smith) (Lepidoptera: Noctuidae), an invasive pest on maize in university jurisdiction. Shivamogga, Karnataka, India: University of Agricultural and Horticultural Sciences,

https://drive.google.com/file/d/1hEW58nhZViHPnRduCj RHVIfWhGASHLSH/view

[118] Sharanabasappa, Kalleshwaraswamy, C. M., Asokan, R., Mahadeva Swamy, H. M., Maruthi, M. S., Pavithra, H. B., Kavita Hedge, Shivaray Navi, Prabhu, S. T., Goergen, G., 2018. First report of the Fall armyworm, Spodoptera frugiperda (J E Smith) (Lepidoptera: Noctuidae), an alien invasive pest on maize in India. Pest Management in Horticultural Ecosystems, 24(1): 23-29.

[119] Shorey HH, Summers CG, Sisk CB, Gerber RG, 1994. Disruption of pheromone communication in Spodoptera exigua (Lepidoptera: Noctuidae) in tomatoes, alfalfa, and cotton. Environmental Entomology, 23(6):1529-1533

[120] Shylesha, A. N., Jalali, S. K., Gupta, A., Varshney, R., Venkatesan, T., Shetty, P., Ojha, R., Ganiger, P. C., Navik, O., Subaharan, K., Bakthavatsalam, N., Ballal, C. R., 2018. Studies on new invasive pest Spodoptera frugiperda (J. E. Smith) (Lepidoptera: Noctuidae) and its natural enemies. Journal of Biological Control, 32(3), 2018.

[121] Sifuentes A JA, 1978. Pests of maize in Mexico and some thoughts on their control. Folleto de Divulgacion, SARH, Instituto Nacional de Investigaciones Agricolas, 58

[122] Silva PSLe, Diniz Filho ET, Granjeiro LC, Duarte SR, 2000. Effects of nitrogen rates and deltamethrin application on yields of green ears and grain yield of maize. (Efeitos de níveis de nitrogênio e da aplicação de deltametrina sobre os rendimentos de espigas verdes e de grãos de milho.) Revista Ceres, 47(269):75-87 
[123] Silva RBda, Cruz I, Figueiredo Mde LC, Bortoni MA, Pereira AG, Melo IFde, Camargo LF, Penteado-Dias $\mathrm{AM}, 2012$. Record of new species of parasitoids on larvae of Spodoptera frugiperda (J. E. Smith) (Lepidoptera: Noctuidae) and Dichomeris famulata Meyrick (Lepidoptera: Gelechiidae) in maize (Zea mays L.) in Brazil. Revista Brasileira de Milho e Sorgo, 11(1):125-129.

http://rbms.cnpms.embrapa.br/index.php/ojs/article/view/ 398

[124] Soares JJ, Silva MS, 2003. Effect of planting date on the production and the ocurrence of pests on cotton (Gossypium hirsutum). (Efeito da época de plantio na produção e na ocorrência de pragas em culturas do algodoeiro (Gossypium hirsutum)). Arquivos do Instituto Biológico (São Paulo), 70(3):295-302. http://www.biologico.sp.gov.br/ARQUIVOS/V70_3/soar es.PDF

[125] Solano Y, Sosa F, Pérez de Camacaro M, 2015. Record of noctuids (Lepidoptera: Noctuidae) associated with strawberry crop in western Venezuela. (Registros de noctuidos (Lepidoptera: Noctuidae) asociados al cultivo de fresa en el occidente de Venezuela.) Entomotropica, 30(19):193-200.

http://www.entomotropica.org/index.php/entomotropica/a rticle/view/488/616

[126] Sparks, A. N., 1979. A review of the biology of the fall armyworm. Florida Entomologist, 62, 82-87. doi: $10.2307 / 3494083$

[127] Starratt AN, McLeod DGR, 1982. Monitoring fall armyworm, Spodoptera frugiperda (Lepidoptera: Noctuidae), moth populations in southwestern Ontario with sex pheromone traps. Canadian Entomologist, 114(7):545-549

[128] Stokstad, E., 2017. New crop pest takes africa at lightning speed. Science, 356(6337), 473-474. https://doi.org/10.1126/science.356.6337.473

[129] Swamy, H. M. M., Asokan, R., Kalleshwaraswamy, C. M., Sharanabasappa, Prasad, Y. G., Maruthi, M. S., Shashank, P. R., Devi, N. I., Anusha Surakasula, Adarsha, S., Srinivas, A., Srinivasa Rao, Vidyasekhar, Raju, M. S., Reddy, G. S. S., Nagesh, S. N., 2018. Prevalence of "R" strain and molecular diversity of fall army worm Spodoptera frugiperda (J.E. Smith) (Lepidoptera: Noctuidae) in India. Indian Journal of Entomology, 80(3), 544-553. http://www.indianjournals.com/ijor.aspx?target=ijor:ije\& type=home doi: 10.5958/0974-8172.2018.00239.0

[130] Todd EL, Poole RW, 1980. Keys and illustrations for the armyworm moths of the noctuid genus Spodoptera Guenee from the Western Hemisphere. Annals of the Entomological Society of America, 73(6):722-738

[131] Togola, A., Meseka, S., Menkir, A., Badu-Apraku, B., Bouka, O., Tamò, M., Djouaka, R., 2018. Measurement of Pesticide Residues from Chemical Control of the Invasive Spodoptera frugiperda (Lepidoptera: Noctuidae) in a Maize Experimental Field in Mokwa, Nigeria. Int. J.
Environ. Res. Public Health, 15, 849. doi: 10.3390/ijerph15050849

[132] Uzayisenga, B., Waweru, B., Kajuga, J., Karangwa, P., Uwumukiza, B., Edgington, S., Thompson, E., Offord, L., Cafá, G., Buddie, A., 2018. First Record of the Fall Armyworm, Spodoptera frugiperda (J.E. Smith, 1797) (Lepidoptera: Noctuidae), in Rwanda. African Entomology, 26(1), 244-246.

[133] Westbrook, J. K., Nagoshi, R. N., Meagher, R. L., Fleischer, S. J., Jairam, S., 2016. Modeling seasonal migration of fall armyworm moths. International Journal of Biometeorology, 60(2), 255-267. http://link.springer.com/article/10.1007\%2Fs00484-0151022-x doi: 10.1007/s00484-015-1022-x

[134] Wu, Q. L., Jiang, Y. Y., Wu, K. M., 2019. Analysis of migration routes of fall armyworm, Spodoptera frugiperda (J. E. Smith) from Myanmar to China. Plant Protection, 45(2), 1-9.

[135] Abrahams P, Bateman M, Beale T, Clottey V, Cock M, Colmenarez Y, Corniani N, Day R, Early R, Godwin J, Gomez J, Gonzalez Moreno P, Murphy ST, OppongMensah B, Phiri N, Pratt C, Silvestri S, Witt A, 2017. Evidence Note, (2), September 2017., Wallingford, UK: CABI.

[136] CABI, Undated. Compendium record. Wallingford, UK: CABI

[137] CABI, Undated a. CABI Compendium: Status as determined by CABI editor. Wallingford, UK: CABI

[138] Chinwada P, 2018. Fall Armyworm Prevalence Assessment in Madagascar. In: FAO Mission Report, 32 pp.

[139] Cock M J W, Beseh P K, Buddie A G, Cafá G, Crozier J, 2017. Molecular methods to detect Spodoptera frugiperda in Ghana, and implications for monitoring the spread of invasive species in developing countries. Scientific Reports. 7 (1), 4103. DOI:10.1038/s41598017-04238-y

[140] Day R, Abrahams P, Bateman M, Beale T, Clottey V, Cock M, Colmenarez Y, Corniani N, Early R, Godwin J, Gomez J, Moreno P G, Murphy S T, Oppong-Mensah B, Phiri N, Pratt C, Silvestri S, Witt A, 2017. Fall armyworm: impacts and implications for Africa. Outlooks on Pest Management. 28 (5), 196-201. http://www.ingentaconnect.com/content/resinf/opm DOI:10.1564/v28_oct_02

[141] Dharmpal Kerketta, Verma L R, Ayam G P, Yadav R S, 2020. First invasive report of fall armyworm, Spodoptera frugiperda (J. E. Smith) (Lepidoptera: Noctuidae) from Orissa, India. Journal of Experimental Zoology, India. 23 (1), 465-468.

[142] Didonet J, Didonet A P P, Erasmo E L, Santos G R dos, 2001. Incidence and population dynamics of pests and their natural enemies in upland rice in Gurupi, Tocantins. (Incidência e densidade populacional de pragas e inimigos naturais em arroz de terras altas, em GurupiTO.). Bioscience Journal. 17 (1), 67-76. 
[143] EPPO, 2019. Spodoptera frugiperda continues to spread in Asia. In: EPPO Reporting Service, Paris, France: EPPO. https://gd.eppo.int/reporting/article-6483

[144] EPPO, 2020. EPPO Global database. In: EPPO Global database, Paris, France: EPPO.

[145] FAO, 2017. Briefing Note on FAP Actions on Fall Armyworm in Africa 15 December 2017., 7 pp. http://www.fao.org/fileadmin/templates/fcc/map/map_of_ affected_areas/Fall_Armyworm_brief__15Dec2017_.pdf FAO, Rome, Italy

[146] FAO, 2017a. FAO Advisory Note on Fall Armyworm (FAW) in Africa. In: FAO Advisory Note on Fall Armyworm (FAW) in Africa. 5 June 2017, Rome, Italy: FAO. 7 pp.

[147] FAO, 2018. Briefing Note on FAO Actions on Fall Armyworm in Africa 31 January 2018. In: Briefing Note on FAO Actions on Fall Armyworm in Africa 31 January 2018, Rome, Italy: FAO. 6 pp.

[148] FAO, 2019. Briefing note on FAO actions on fall armyworm., Rome, Italy: FAO. http://www.fao.org/3/abs183e.pdf

[149] FAO, 2019a. FAO Statement on Fall Armyworm in Sri Lanka., Rome, Italy: FAO. http://www.fao.org/srilanka/news/detailevents/en/c/1177796/

[150] FAO, 2019b. FAW Briefing Note July 2019., Rome, Italy: FAO.

[151] FAO, 2019c. First Detection of Fall Armyworm in China., Rome, Italy: FAO. https://www.ippc.int/fr/news/first-detection-of-fallarmyworm-in-china/

[152] FAO, 2019d. Food Chain Crisis Early Warning Bulletin., Rome, Italy: FAO. http://www.fao.org/3/ca5487en/ca5487en.pdf

[153] Ganiger PC, Yeshwanth HM, Muralimohan K, Vinay N, Kumar ARV, Chandrashekara K, 2018. Occurrence of the new invasive pest, fall armyworm, Spodoptera frugiperda (J.E. Smith) (Lepidoptera: Noctuidae), in the maize fields of Karnataka, India. In: Current Science, 115 (4) 621 623.

[154] Greathead D J, Greathead A H, 1992. Biological control of insect pests by insect parasitoids and predators: the BIOCAT database. Biocontrol News and Information. 13 (4), $61 \mathrm{~N}-68 \mathrm{~N}$

[155] Gurrola-Pérez C C, Álvarez-Zagoya R, HernándezMendoza J L, Correa-Ramírez M, Pérez-Santiago G, 2018. Record of Lespesia archippivora, Lespesia postica, and Archytas marmoratus parasitizing larvae of Spodoptera frugiperda in Durango, Mexico. (Registro de Lespesia archippivora, Lespesia postica, y Archytas marmoratus Parasitando larvas de Spodoptera frugiperda en Durango, México.). Southwestern Entomologist. 43 (2), 505-510. http://www.bioone.org/loi/swen DOI:10.3958/059.043.0221

[156] Huis A van, 1981. Integrated pest management in the small farmer's maize crop in Nicaragua. In: Mededelingen Landbouwhogeschool Wageningen, 81 (6) $221 \mathrm{pp}$.
[157] ICAR-NBAIR, 2018. PEST ALERT: 30th July, 2018. Spodoptera frugiperda (Smith, J.E.) (Insecta: Lepidoptera), $\quad$ India: ICAR-NBAIR. http://www.nbair.res.in/recent_events/Pest\%20Alert\%20 30th\%20July\%202018-new1.pdf

[158] ICAR-NBAIR, 2018a. Spodoptera frugiperda (J. E. Smith). Insects in Indian Agrosystems., India: ICARNational Bureau of Agricultural Insect Resources (NBAIR).

http://www.nbair.res.in/insectpests/Spodoptera_frugiperd a.php

[159] IITA, 2016. First report of outbreaks of the "Fall Armyworm" on the African continent. In: IITA Bulletin, No. 2330, http://bulletin.iita.org/index.php/2016/06/18/first-reportof-outbreaks-of-the-fall-armyworm-on-the-africancontinent/

[160] IITA, 2018. Fall armyworm has reached the Indian subcontinent!, Ibadan, Nigeria: IITA. http://www.iita.org/news-item/fall-armyworm-hasreached-the-indian-subcontinent/

[161] IPAPEL-FAO, 2017. (Rapport de mission d'évaluation de l'incidence de l'attaque de la chenille Spodoptera sp. et prélèvement des échantillons de la chenille dans les territoires de Kambove et de Pweto à kilwa dans la Province du Haut Katanga du 07 au 10 février 2017. FAO and)., Democratic Republic of Congo: Université de Lubumbashi.

[162] IPPC, 2016. (Les dégâts causés par Spodoptera frugiperda). In: IPPC Official Pest Report, Rome, Italy: FAO. https://www.ippc.int/

[163] IPPC, 2017. Detection of Fall Army Worm Spodoptera frugiperda in Swaziland. In: IPPC Official Pest Report, Rome, Italy: FAO. https://www.ippc.int/en/

[164] IPPC, 2017a. IPPC Official Pest Report., Rome, Italy: FAO. https://www.ippc.int/en/

[165] IPPC, 2017b. IPPC Official Pest Report., Rome, Italy: FAO. https://www.ippc.int/en/

[166] IPPC, 2017c. IPPC Official Pest Report., Rome, Italy: FAO. https://www.ippc.int/en/

[167] IPPC, 2018. Report on Fall armyworm (Spodoptera frugiperda). Rome, Italy: FAO. https://www.ippc.int/

[168] IPPC, 2019. (Signalement de la chenille légionnaire d'automne (CLA) au Gabon). In: IPPC Official Pest Report, Rome, Italy: FAO. https://www.ippc.int/en/

[169] IPPC, 2019a. First Detection Report of the Fall Armyworm Spodoptera frugiperda (Lepidoptra: Noctuidae) on Maize in Myanmar. In: IPPC Official Pest Report, Rome, Italy: FAO. https://www.ippc.int/en/

[170] IPPC, 2019b. Report of first detection of Fall Armyworm(FAW) in Republic of Korea. In: IPPC Official Pest Report, Rome, Italy: FAO. https://www.ippc.int/en/

[171] IPPC, 2019c. Report of first detection of Spodoptera frugiperdea - Fall Armyworm (FAW) in Japan. In: IPPC Official Pest Report, Rome, Italy: FAO. https://www.ippc.int/en/ 
[172] IPPC, 2019d. Report of first detection of Spodoptera frugiperda - Fall Armyworm (FAW) in Egypt. In: IPPC Official Pest Report, Rome, Italy: FAO. https://www.ippc.int/en/

[173] IPPC, 2019e. Reporting Fall Armyworm (Spodoptera frugiperda) in Yemen. In: IPPC Official Pest Report, Rome, Italy: FAO. https://www.ippc.int/en/

[174] IPPC, 2019f. Spodoptera frugiperda (Fall Armyworm). In: IPPC Official Pest Report, Rome, Italy: FAO. https://www.ippc.int/en/

[175] IPPC, 2019g. The Occurence of Fall Armyworm (Spodoptera frugiperda) in Indonesia. In: IPPC Official Pest Report, Rome, Italy: FAO. https://www.ippc.int/en/

[176] IPPC, 2020. First detection of Spodoptera frugiperda (fall armyworm) in Torres Strait., Rome, Italy: FAO. https://www.ippc.int/

[177] IPPC, 2020a. The first detection of Fall armyworm (FAM), Spodoptera frugiperda, in United Arab Emirates. In: IPPC Official Pest Report, No. ARE-01/1, Rome, Italy: FAO. https://www.ippc.int/

[178] IPPC, 2020b. Report of first detection of Fall Armyworm (FAW) in 2020 of Republic of Korea. In: IPPC Official Pest Report, Rome, Italy: FAO. https://www.ippc.int/

[179] IPPC, 2020c. Further detections of Spodoptera frugiperda (fall armyworm) on mainland Australia. In: IPPC Official Pest Report, Rome, Italy: FAO. https://www.ippc.int/

[180] IPPC, 2020d. Report on Fall armyworm (Spodoptera frugiperda). In: IPPC Official Pest Report, Rome, Italy: FAO. https://www.ippc.int/

[181] Jacobs A, van Vuuren A, Rong IH, 2018. Characterisation of the fall armyworm (Spodoptera frugiperda J.E. Smith) (Lepidoptera: Noctuidae) from South Africa. In: African Entomology, 26 (1) 45-49.

[182] Köppen, W. Die Wärmezonen der Erde, nach der Dauer der heissen, gemässigten und kalten Zeit und nach der Wirkung der Wärme auf die organische Welt betrachtet. Meteorologische Zeitschrift 1, 215-226 (1884).

[183] Lee GwanSeok, Seo BoYoon, Lee JongHo, Kim HyunJu, Song JeongHeub, Lee WonHoon, 2020. First report of the fall armyworm, Spodoptera frugiperda (Smith, 1797) (Lepidoptera, Noctuidae), a new migratory pest in Korea. Korean Journal of Applied Entomology. 59 (1), 73-78.

[184] Martel P, Hudon M, Ritchot C, 1980. The incidence of insect pests in certain crops in the south-west of Quebec in 1979. (Etat des insectes nuisibles dans certaines cultures du sud-ouest du Quebec en 1979.). Annals of the Entomological Society of Quebec. 25 (3), 190-194.

[185] Ministry of Agriculture of the State of Eritrea, 2018. Eritrea: Crops are safe from Fall Army Worm infestation. In: Fall Army Worm Newsletter, https://www.ippc.int/static/media/files/countrynews/2018 /08/10/FAW_Newsletter_August.pdf

[186] Naeem-Ullah U, Ashraf Ansari M, Iqbal N, Saeed S, 2019. First authentic report of Spodoptera frugiperda (J.E. Smith) (Noctuidae: Lepidoptera) an alien invasive species from Pakistan. Applied Sciences and Business Economics. 6 (1), 1-3. http://www.asbejournal.org/wp-
content/uploads/2019/09/1_First_authentic_report_of_Sp odoptera_frugiperda_J.E.SmithNoctuidae_Lepidoptera_a n_alien_invasive_species_from_Pakistan_ASBE_v6_i1_ p1_2019.pdf

[187] NPPO of the Netherlands, 2013. Pest status of harmful organisms in the Netherlands., Wageningen, Netherlands:

[188] Otim M H, Tay W T, Walsh T K, Kanyesigye D, Adumo S, Abongosi J, Ochen S, Sserumaga J, Alibu S, Abalo G, Asea G, Agona A, 2018. Detection of sister-species in invasive populations of the fall armyworm Spodoptera frugiperda (Lepidoptera: Noctuidae) from Uganda. PLoS ONE. $\quad 13 \quad$ (4), e0194571. http://journals.plos.org/plosone/article?id=10.1371/journa 1.pone.0194571 DOI:10.1371/journal.pone.0194571

[189] Pratissoli D, Polanczyk R A, Grecco E D, Ferreira R A, Holtz A M, 2007. Microbial toxicity of new Bacillus thuringiensis isolates in two populations of Spodoptera frugiperda originating from Minas Gerais and Espírito Santo. (Efeito entomotóxico de novos isolados de Bacillus thuringiensis em duas populações de Spodoptera frugiperda oriundas de Minas Gerais e do Espírito Santo.). Revista Brasileira de Milho e Sorgo. 6 (2), 140148.

[190] Queensland Government Department of Agriculture and Fisheries, 2020. First mainland detection of fall armyworm., Australia: Queensland Government Department of Agriculture and Fisheries. https://www.daf.qld.gov.au/news-media/mediacentre/biosecurity/news/first-mainland-detection-of-fallarmyworm

[191] Rambajan I, 1981. Major insect pests of paddy in Guyana. International Rice Research Newsletter. 6 (6), 16-17.

[192] Ratna B A S, Binu B, Premnidhi S, Pathour R S, Naresh M M, Raza H T, 2019. First record of fall army worm Spodoptera frugiperda (J. E. Smith) from Nepal. Indian Journal of Entomology. 81 (4), 635-639.

[193] Sharanabasappa, Kalleshwara swamy CM, 2018. Presence of fall armyworm, Spodoptera frugiperda (J E Smith) (Lepidoptera: Noctuidae), an invasive pest on maize in university jurisdiction., Shivamogga, Karnataka, India: University of Agricultural and Horticultural Sciences.

https://drive.google.com/file/d/1hEW58nhZViHPnRduCj RHVIfWhGASHLSH/view

[194] Sharanabasappa, Kalleshwaraswamy C M, Asokan R, Swamy H M M, Maruthi M S, Pavithra H B, Kavita Hegde, Shivaray Navi, Prabhu S T, Goergen G, 2018. First report of the fall armyworm, Spodoptera frugiperda (J E Smith) (Lepidoptera: Noctuidae), an alien invasive pest on maize in India. Pest Management in Horticultural $\begin{array}{llll}\text { Ecosystems. } & 24 & \text { (1), } & \text { 23-29. }\end{array}$ http://aapmhe.in/index.php/pmhe/article/view/816

[195] Sifuentes A J A, 1978. Pests of maize in Mexico and some thoughts on their control. (Plagas del maiz en Mexico y algunas consideraciones sobre su control.). In: Folleto de Divulgacion, SARH, Instituto Nacional de Investigaciones Agricolas, $30 \mathrm{pp}$. 
[196] Silva P S L e, Diniz Filho E T, Granjeiro L C, Duarte S $R, 2000$. Effects of nitrogen rates and deltamethrin application on yields of green ears and grain yield of maize. (Efeitos de níveis de nitrogênio e da aplicação de deltametrina sobre os rendimentos de espigas verdes e de grãos de milho.). Revista Ceres. 47 (269), 75-87.

[197] Soares J J, Silva M S, 2003. Effect of planting date on the production and the ocurrence of pests on cotton (Gossypium hirsutum). (Efeito da época de plantio na produção e na ocorrência de pragas em culturas do algodoeiro (Gossypium hirsutum).). Arquivos do Instituto Biológico (São Paulo). $70 \quad$ (3), 295-302. http://www.biologico.sp.gov.br/ARQUIVOS/V70_3/soar es.PDF

[198] Solano Y, Sosa F, Pérez de Camacaro M, 2015. Record of noctuids (Lepidoptera: Noctuidae) associated with strawberry crop in western Venezuela. (Registros de noctuidos (Lepidoptera: Noctuidae) asociados al cultivo de fresa en el occidente de Venezuela.). Entomotropica. 30 (19), 193-200. http://www.entomotropica.org/index.php/entomotropica/a rticle/view/488/616

[199] Starratt A N, McLeod D G R, 1982. Monitoring fall armyworm, Spodoptera frugiperda (Lepidoptera: Noctuidae), moth populations in southwestern Ontario with sex pheromone traps. Canadian Entomologist. 114 (7), 545-549.

[200] Swamy H M M, Asokan R, Kalleshwaraswamy C M, Sharanabasappa, Prasad Y G, Maruthi M S, Shashank P R, Devi N I, Anusha Surakasula, Adarsha S, Srinivas A, Srinivasa Rao, Vidyasekhar, Raju M S, Reddy G S S, Nagesh S N, 2018. Prevalence of "R" strain and molecular diversity of fall army worm Spodoptera frugiperda (J.E. Smith) (Lepidoptera: Noctuidae) in India. Indian Journal of Entomology. 80 (3), 544-553. http://www.indianjournals.com/ijor.aspx?target=ijor:ije\& type=home DOI:10.5958/0974-8172.2018.00239.0

[201] Uzayisenga B, Waweru B, Kajuga J, Karangwa P, Uwumukiza B, Edgington S, Thompson E, Offord L, Cafá G, Buddie A, 2018. First Record of the Fall Armyworm, Spodoptera frugiperda (J.E. Smith, 1797) (Lepidoptera: Noctuidae), in Rwanda. In: African Entomology, 26 (1) 244-246. 